\title{
THE ELUCIDATION OF MECHANISMS IN HOMOGENEOUS CATALYSIS
}

\section{RINALDO POLI}

CNRS; Laboratoire de Chimie de Coordination; Université de Toulouse; UPS, INPT; Toulouse, France and Institut Universitaire de France, 103, bd Saint-Michel, 75005 Paris, France

\begin{abstract}
After a reminder of the definition of common terms used in catalysis and reaction mechanisms, different chapters highlight the various approaches used for the elucidation of catalytic mechanisms, each with its own advantages and limitations. Kinetics investigations define the rate law and provide information on certain features of the reaction pathway between the resting state and the transition state of the rate-determining step. The use of isotope labels is useful in three ways: through the measurement of kinetic isotope effects, through the study of the incorporation into the products (regio-, stereochemical features), and through the incorporation into the incompletely converted reactants (reversibility). The investigation of isolated intermediates, if available, gives experimental structural information and thermodynamic/activation data on selected stoichiometric steps of the catalytic cycle. In situ spectroscopic investigations help determine the chemical nature of the resting state and other low energy intermediates if carried out under catalytic conditions, or assist in the identification of other intermediates if performed under modified conditions on stoichiometric steps. Effects of the ligand modification on catalytic activity and selectivity also provide information on kinetically relevant steps. Finally, the application of computational methods is highlighted, with emphasis on the advantages and limitations of different computational strategies, the choice of basis functions and chemical model, and the handling of solvent models for the computation of thermodynamically and kinetically relevant parameters in solution.
\end{abstract}

Address correspondence to Rinaldo Poli, Laboratoire de Chimie de Coordination, 205, route de Narbonne, F-31077 Toulouse, France. E-mail : rinaldo.poli@1cc-toulouse.fr. 
SUMMARY

1. INTRODUCTION 3

2. A FEW DEFINITIONS 4

3. KINETICS 11

4. ISOTOPE LABELLING STUDIES 16

$\begin{array}{ll}\text { 4.1 Kinetic isotope effects } & 17\end{array}$

4.2 Distribution of the isotope labels in the products 23

4.3 Reversibility of substrate activation steps 25

5. STOICHIOMETRIC STUDIES ON ISOLATED INTERMEDIATES 27

6. IN SITU SPECTROSCOPIC INVESTIGATIONS 29

6.1 In situ investigations of a catalytic run 29

6.2 Investigating the identity of missing intermediates 35

7. EFFECTS OF THE LIGAND STRUCTURE 37

8. THEORETICAL CALCULATIONS 38

8.1 Computational methods 41

$\begin{array}{ll}\text { 8.2 Basis functions } & 47\end{array}$

8.3 Thermodynamic properties, chemical and solvent model 51

9. CONCLUSION 56

10. ACKNOWLEDGEMENT 57

11. REFERENCES 57 


\section{INTRODUCTION}

In this «technique » article, we address the complex topic of how one approaches the deduction of mechanisms in homogeneous catalysis. The article will not provide a cookbook recipe that can be applied to every situation. This is impossible. The study of reaction mechanisms in general and of catalytic mechanisms in particular is haunted by numerous difficulties as will be pointed out in due course and each reaction or catalytic cycle has its own specificities. The article is just meant to introduce various different and complementary approaches that have the potential of providing useful information toward the ultimate elucidation of a catalytic mechanism. Indeed, it normally takes the combination of many different approaches to arrive at a reasonably complete understanding of a catalytic mechanism. There is a popular statement that a reaction mechanism can never be proven, whereas a wrong mechanistic hypothesis may be ruled out if the results of suitable experiments that have been set out to test it are contrary to expectations. The correct mechanism will, of course, be in agreement with the results of any correctly carried out experiment. Thus, it is possible to arrive at a reasonable confidence in favour of a certain mechanism when all possible alternatives have been ruled out. Nowadays, with the help of highly sensitive in situ characterization techniques, the appropriate use of isotope labels and high-level computations, in combination with kinetics studies and stoichiometric investigations of elementary steps involving real intermediates and model compounds, our confidence in a mechanism can reach very high levels, approaching $100 \%$ in certain cases. It is therefore necessary to be familiar with all the available approaches for the elucidation of mechanisms and with the criteria that help us select the appropriate experiment for a given situation. The article is divided into chapters devoted to the various available approaches. Comprehensive reviews on this specific subject do not seem to be available. Many books and 
monographs are devoted to catalysis itself but only few address the methods used for investigating catalytic mechanisms. ${ }^{[1,2]}$ Selected approaches are presented in a few other sources. ${ }^{[3-6]}$ Other literature references will be cited within each chapter. The article is based on a tutorial delivered in Camerino (Italy) on 23 August 2009 within the framework of an "Intensive Programme" on "Advanced Catalysis and Organometallic Chemistry" for students at the Masters and Doctoral level.

\section{A FEW DEFINITIONS}

Before starting with the analysis of the various available approaches toward the elucidation of catalytic mechanisms, it is useful to remind ourselves of the meaning of a certain number of terms. A reaction mechanism is the step by step sequence of elementary reactions, each of which is defined as a direct transformation of one or more reactant molecules into one or more product molecules through a single transition state, as shown schematically in Figure $1 a$. The energy profile thus relates two local minima through a single maximum on the potential energy surface (PES). It may be represented on an energy, enthalpy, or free energy scale. The energy required to take the system from the reactants state to transition state is the threshold energy $\Delta E^{\star}$ (or enthalpy $\Delta H^{*}$, free energy $\Delta G^{\ddagger}$ ), also usually referred to as activation energy (or enthalpy, free energy). Strictly speaking, use of the term "activation energy" should be restricted to the empirical parameter $E_{\text {act }}$ characterizing the exponential temperature dependence of the rate constant of the elementary reaction (Arrhenius relationship, equation 1). The threshold (or activation) enthalpy and free energy are related to the rate constant though the Eyring relationship, derived from transition state theory (equation 2). 

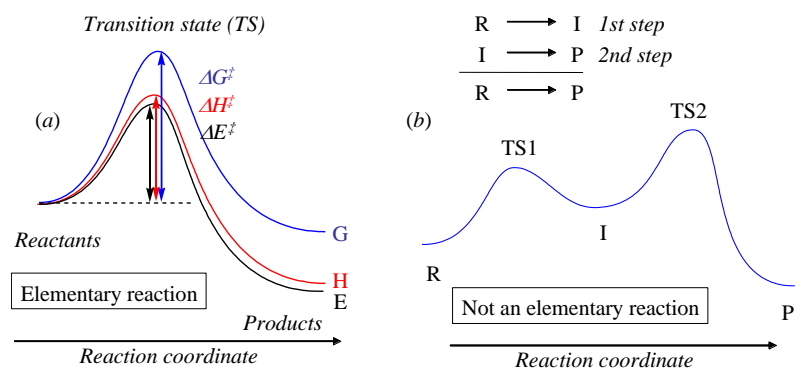

Figure 1. Energetic profile of (a) an elementary reaction; (b) a two-step process.

$$
\begin{aligned}
& k=A e^{-\frac{E_{c c t}}{R T}} \\
& \ln \left(\frac{k h}{k_{B} T}\right)=-\frac{\Delta H^{\ddagger}}{R T}+\frac{\Delta S^{\ddagger}}{R}=-\frac{\Delta G^{\ddagger}}{R T}
\end{aligned}
$$

Elementary reactions are classified according to their molecularity, which corresponds to the number of reactant molecules undergoing the chemical transformation. Thus unimolecular reactions are those where a single molecule reacts (as in a bond cleavage or isomerization step), a bimolecular reaction involves the encounter and subsequent transformation of two molecules, and so forth. Termolecular reactions as well as reactions of higher order are extremely rare. According to collision theory, a unimolecular reaction also requires a collision of the reacting molecule with a neutral body (spectator molecules such as the solvent, or the vessel walls) giving it enough extra energy to overcome the reaction barrier. The molecularity of an elementary reaction determines its rate law (the order of the reaction is 1 for each reacting partner). Figure $1 b$ shows the mechanism for a generic two-step reaction. The rate law in this case is not necessarily related to the molecularity of any of its elementary steps (vide infra, chapter on kinetics).

In the mechanism of a catalyzed transformation, the catalyst is regenerated at the end of the process, thus this may be represented by a sequence of elementary steps in a cycle, generally referred to as catalytic cycle, as shown for instance in Figure $2 a$. Knowledge of the 
mechanism implies that the sequence of elementary steps and the identity of each participating species is perfectly defined. The representation as a cycle is equivalent to that as a sequence of elementary transformations (Figure $2 b$ ). Another useful representation is as the energy profile, for instance on a free energy scale (Figure $2 c$ ), which contains additional information about the reaction barriers of each elementary step - $\Delta \mathrm{G}_{1}^{\star}, \Delta \mathrm{G}_{2}^{\ddagger}, \ldots$ - as well as the thermodynamic parameters associated to each step and to the overall transformation $\Delta \mathrm{G}_{\mathrm{rxn}}$.

(a)

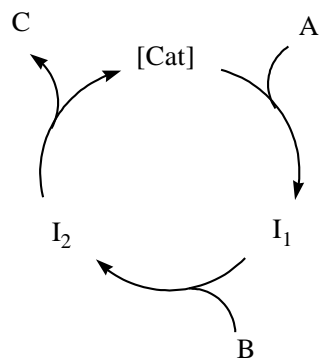

(b)

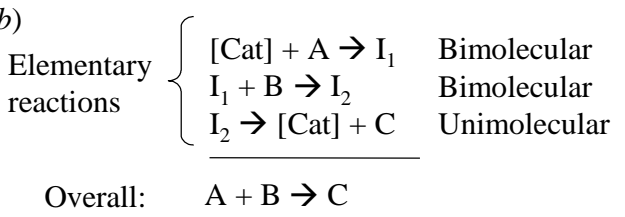

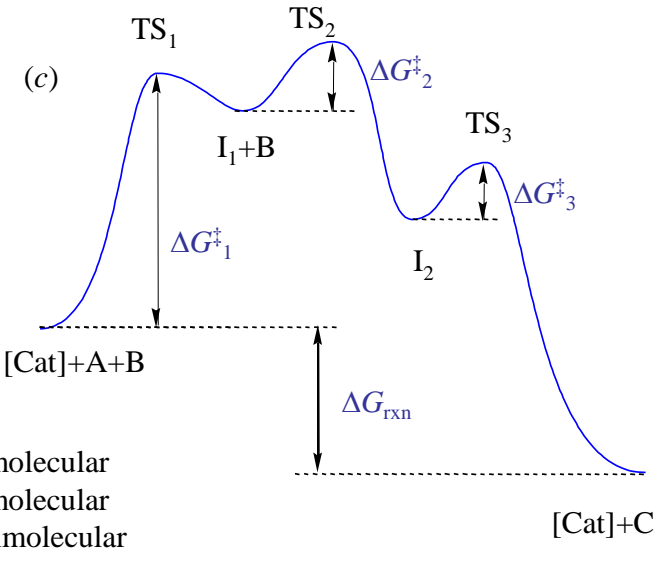

Figure 2. Representation of a catalytic cycle in which two reagents A and B are transformed into a product $\mathrm{C}$ by a catalytic species [Cat] and three elementary steps through the two intermediates $\mathrm{I}_{1}$ and $\mathrm{I}_{2}$.

A pre-catalyst is a stable compound that is used to generate the active catalyst in situ by some sort of activation process. Occasionally one or more of the different species implicated in the catalytic cycle are sufficiently stable to be stored and used to start the catalytic process. An off-loop species is defined as a complex in equilibrium with one or more of the catalytic intermediates but not involved itself in the catalytic cycle. Its free energy may be higher or lower than that of the intermediate in direct equilibrium with it. Thus, certain off-loop species may actually be isolable compounds and may be used to start the process. However, if the 
equilibrium is too displaced toward this compound, then its formation is irreversible and it represents a catalyst deactivation process and the compound produced can be considered as a dead catalyst (Figure 3 ).

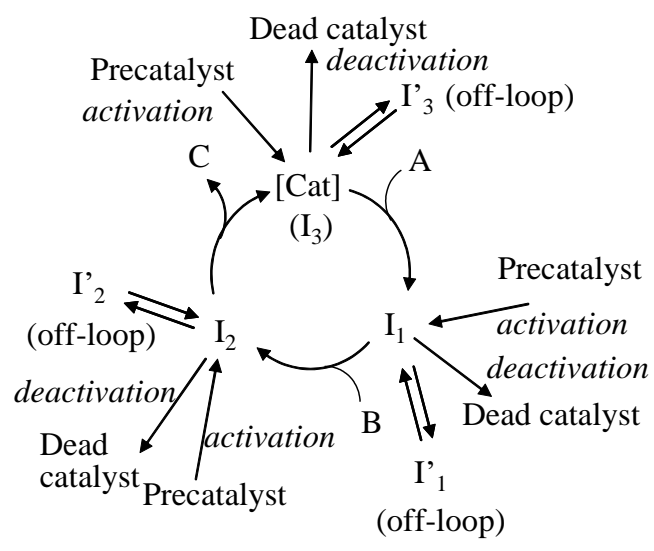

Figure 3. Pre-catalysts, off-loop species, catalytic intermediates and dead catalysts.

When we represent multiple cycles on an energy profile, for instance two as in Figure 4, it is obvious that species [Cat] has no special property distinguishing it from the other catalytic intermediates. Any of the catalytic intermediates may be generated from a stable precatalyst and be in equilibrium with off-loop species. The question then arises: which species is the "catalyst" for a given cycle? The answer is: any of the species capable of generating the catalytic process if placed under catalytic conditions, namely any of the intermediates and any of the off-loop species. All species corresponding to local energy minima along the catalytic cycle may be considered as intermediates and all of them represent different forms of the catalyst. Figure 4 emphasizes the obvious fact that the free energy difference between any intermediate and the same intermediate in the subsequent cycle corresponds to the free enthalpy of the stoichiometric reaction, $\Delta \mathrm{G}_{\mathrm{rxn}}$. 


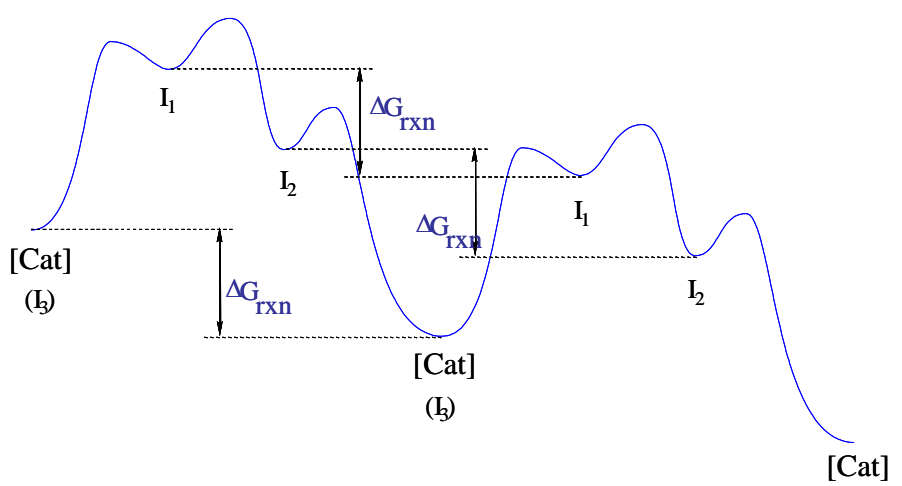

(b)

Figure 4. A generic representation of the energy profile for two subsequent catalytic cycles.

The rate-determining step (RDS) is the slowest step in the catalytic cycle. Its correct identification is an important point in the elucidation of a catalytic mechanism and this is not always a straightforward exercise. In a simple case, it can be identified as the step associated to the highest free-energy transition state in the cycle. Nota bene: this is not necessarily the step with the greatest activation barrier, as shown by the specific example of Figure $2 c$ where the highest free-energy TS is that of step 2 whereas the greatest barrier is associated to step 1. However, there are trickier cases such as that shown in Figure 5. Which step is rate determining? An unambiguous way to identify the RDS is as follows. Each local minimum can be related to the next one by a positive (up) or a negative (down) $\Delta G$ change. For a set of three consecutive intermediates, the possible sequences are down-up, down-down, up-up and up-down. We first need to identify all the catalytic intermediates that have a $G$ lower than both the preceding and the following intermediate, namely the down-up sequences (in the example of Figure 5, we can find two, $\mathrm{I}_{4}$ and $\mathrm{I}_{2}$ ). Next, we need to consider only those "stable" intermediates that are in a decreasing free energy order (in Figure 5, both $\mathrm{I}_{4}$ and $\mathrm{I}_{2}$ verify this requirement because $\mathrm{I}_{2}$ has a lower $\mathrm{G}$ than the preceding $\mathrm{I}_{4}$ and a higher one than the subsequent $\mathrm{I}_{4}$ ). Now, we need to locate the highest free energy TS between each "stable" intermediate and the next one. In Figure 5, we find $\mathrm{TS}_{2}$ (between $\mathrm{I}_{4}$ and $\mathrm{I}_{2}$ ) and $\mathrm{TS}_{4}$ (between $\mathrm{I}_{2}$ and $\mathrm{I}_{4}$ ). At this point we need to compare the free energy differences on going from the 
stable intermediate to the high-energy TS on the way to the next stable intermediate [in the present example, $G\left(\mathrm{TS}_{2}\right)-G\left(\mathrm{I}_{4}\right)$ and $\left.G\left(\mathrm{TS}_{4}\right)-G\left(\mathrm{I}_{2}\right)\right]$. The highest of these differences determines the rate determining step. In the example of Figure 5, the highest energy difference relates $\mathrm{I}_{2}$ with $\mathrm{TS}_{4}$, thus the RDS is step 4 , leading from intermediate $\mathrm{I}_{3}$ to intermediate $\mathrm{I}_{4}$ through transition state $\mathrm{TS}_{4}$. Note that, in this example, $\mathrm{TS}_{4}$ has in fact a lower $G$ than the transition state $\mathrm{TS}_{2}$ that precedes it, but a higher one relative to the $\mathrm{TS}_{2}$ of the subsequent cycle. If we focus only on a single cycle starting and ending with $\mathrm{I}_{4}$, the highest $G$ TS is in fact that of step 2, which is not the RDS.

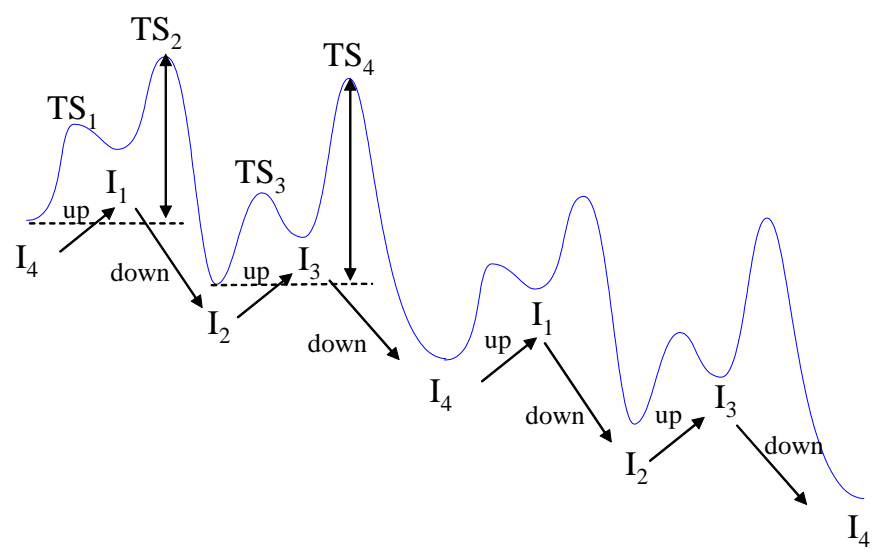

Figure 5. Determination of the rate-determining step and resting state. Example of the free energy profile for two cycles of a four-step catalytic process.

Another important definition for a catalytic cycle is that of resting state. This is simply the state in which the catalyst spends most of the time during the catalyzed reaction. Consequently, it is the state which is present at the highest concentration among all the catalytically active species. The assignment of this species to the intermediate having the lowest free energy is not sufficient, at least for irreversible cycles, once again because the point representing the beginning and end of the cycle is arbitrary. However, the assignment is straightforward once the RDS has been identified. For instance, taking the example of Figure 5 , species $I_{1}$ and $I_{3}$ can immediately be discarded because they have a higher free energy than 
both the preceding and the following intermediates. Amongst intermediates $I_{2}$ and $I_{4}$, the former is transformed into the latter at a slower rate than the latter is transformed into the former. Thus, $I_{2}$ will be the most populated state under catalytic conditions. The resting state can be unequivocally defined as the species having the lowest $\mathrm{G}$ between two consecutive transition states of the RDS. Note that an off-loop species, when it has lower G than the catalytic intermediate in equilibrium with it, may also correspond to the catalyst resting state. In the example shown in Figure 6, the resting state would have been $\mathrm{I}_{1}$ if the $\mathrm{I}_{3} / \mathrm{I}_{3}$ equilibrium were not present.
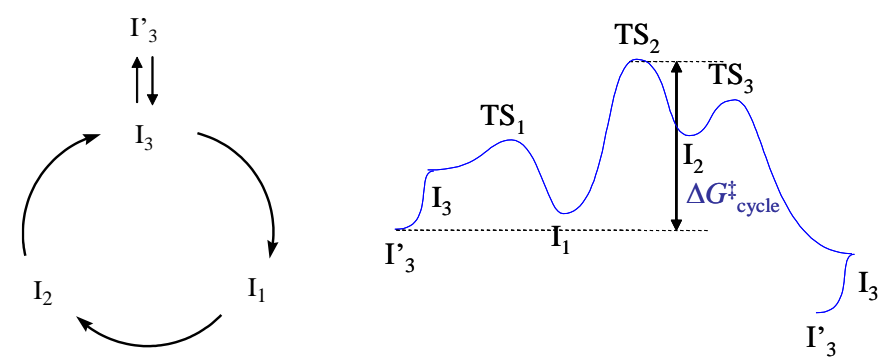

Figure 6. Example of an off-loop species as resting state.

These definitions are important, because the kinetics of the catalytic process are determined only by the events occurring between the resting state and the TS of the RDS (unless reversibility is involved), and because the relative concentrations of the various intermediates (of importance for their in situ detection) are determined by their $G$ relative to the resting state. The catalytic activity, as defined by the turnover frequency (TOF), is related by the Eyring equation to the free energy difference between the TS of the RDS and the resting state $\left(\Delta G^{\ddagger}\right.$ cycle $\left.=G_{\mathrm{TS}(\mathrm{RDS})}-G_{\mathrm{RS}}\right)$, as shown in equation 3. Improvement of the catalytic activity requires lowering $\Delta G^{\ddagger}$ cycle, which can be accomplished either by lowering $G_{\mathrm{TS}(\mathrm{RDS})}$ or by raising $G_{\mathrm{RS}}$. 


$$
T O F=\left(\frac{k_{B} T}{h}\right) e^{-\frac{\Delta G^{\ddagger} \text { cycle }}{R T}}
$$

\section{KINETICS}

The form of the rate law provides useful information on the involvement (or lack thereof) of each substrate in kinetically relevant elementary steps, as well as on their reversibility. The relationship between the form of the rate law and the mechanism is amply discussed in all kinetics textbooks. We shall provide here only a rapid outline of the most relevant relationships. The rate law always shows a non-zero reaction order in catalyst, since a catalytic intermediate is necessarily involved in the RDS, and in one or more of the substrates. The product of the reaction may also appear in the rate expression for reversible cycles (e.g. when $\Delta G_{\mathrm{rxn}}$ is small). The most useful kinetics expressions are those obtained under conditions of irreversibility. The back transformation is essentially absent and can be neglected for any process (even when $\Delta G_{\mathrm{rxn}}=0$ ) for degrees of conversion lower than $5-10 \%$. It can also be neglected at higher degrees of conversion for the limiting reagent if the process is carried out with a large excess of the other one(s). A case where excess of a reagent makes the process practically irreversible, for instance, is the transfer hydrogenation of any ketone ArCOR by isopropanol, leading to acetone and to the alcohol $\operatorname{ArCH}(\mathrm{OH}) \mathrm{R}$, when the reaction is carried out in isopropanol itself as a solvent. The process is practically irreversible under any experimental condition when $\Delta \mathrm{G}_{\mathrm{rxn}}<-5 \mathrm{kcal} / \mathrm{mol}$.

A kinetics investigation of a catalytic process typically involves monitoring of any of the reactants and/or products by any suitable physical technique (spectroscopy, gasvolumetry, pressure changes, etc.), and not the monitoring of the catalytic species, the concentration of which remains approximately constant during the transformation. The 
timescale of the experiment is typically in the range of minutes to hours, suitable for classical mixing and monitoring. In addition to these investigations, however, specific stoichiometric steps of the catalytic cycle may also be the focus of kinetics investigations, if the desired intermediate is available as an isolated pure product (see section 5). In that case, the study may involve the direct monitoring of the catalytic metal complexes and the transformation may occur on a much shorter timescale, requiring special methodologies (stopped-flow rapid mixing techniques, or line-broadening investigations e.g. in NMR spectra).

Before embarking in a kinetics investigation of a catalytic process, a certain number of requirements must be met. One, as mentioned above, is to select conditions where the transformation is irreversible (the back-reaction can be neglected). A second one is to verify that the rate of the transformation is not limited by the rate of the pre-catalyst activation (absence of an induction time). A good example is provided by the hydrogenation of Z-methyl 3-acetamido butenoate catalyzed by $\left[\mathrm{Rh}(\operatorname{cod})_{2}\right] \mathrm{BF}_{4} / \mathrm{Et}$-DuPHOS in methanol. ${ }^{[7]}$ As shown in Figure 7 , the catalytic intermediate under these conditions is complex [Rh(EtDuPHOS $\left.)(\mathrm{MeOH})_{2}\right]^{+}$, obtained in two steps from the pre-catalyst by hydrogenation of the cyclooctadiene ligands. The three cationic rhodium complexes shown in Figure 7 are available as tetrafluoroborate salts, but only the gas absorption data obtained when using the catalytic intermediate allow a straightforward kinetics analysis. Observation of the same enantiomeric excess for the three catalytic runs confirms that the same catalyst operates in all cases. A third requirement is that the process does not suffer from catalyst deactivation. This can be checked in a number of ways. A first one is to verify that the limiting substrate is completely consumed (or that the system reaches equilibrium for reversible processes). A second, more stringent check is to recover and recycle the catalyst (or simply add fresh substrate at the end of the process) and to verify that the catalyzed transformation is resumed 
at the same TOF. The subsequent successful fit of the kinetics data to a rate law confirms the absence of deactivation.
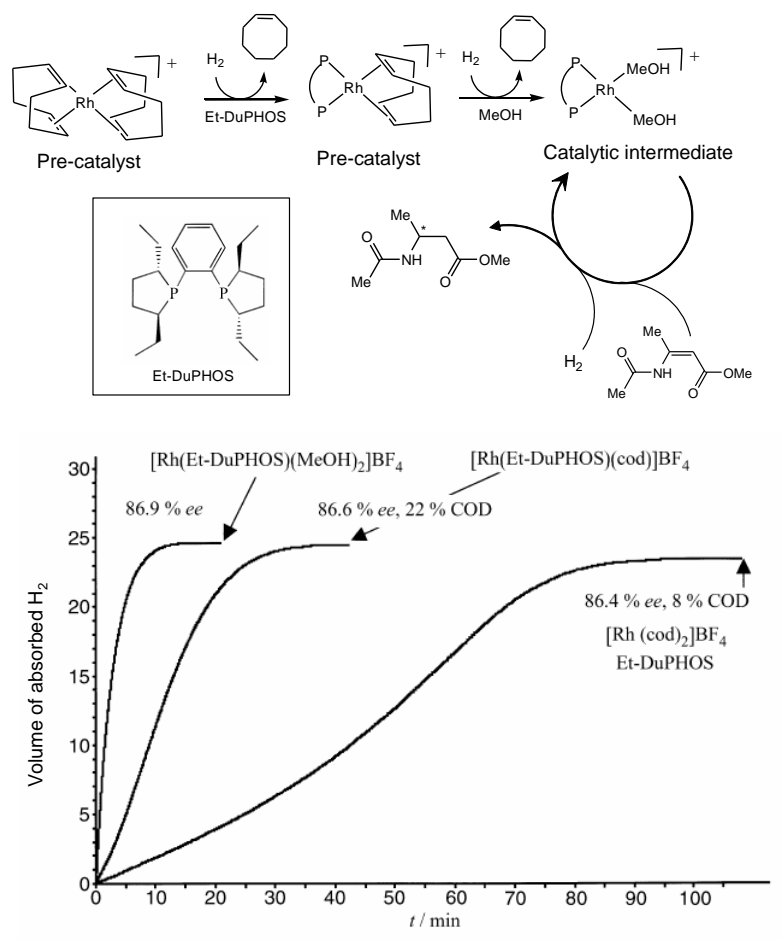

Figure 7. Activation of $\left[\mathrm{Rh}(\operatorname{cod})_{2}\right] \mathrm{BF}_{4} / \mathrm{Et}-\mathrm{DuPHOS}$ in hydrogenation catalysis at $25^{\circ} \mathrm{C}$ and 1 bar of total pressure. ${ }^{[7]}$

The direct availability of a catalytic intermediate as an isolated species is rarely possible, so the rate law expresses the reaction order in pre-catalyst. Since the catalyst does not change concentration during a catalytic run (unless slow pre-catalyst activation or deactivation are involved), analysis of the kinetics data yields an observed rate constant, $k_{\mathrm{obs}}=$

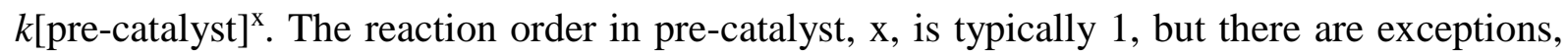
therefore checking is necessary. An example is provided by Figure 8, where a dinuclear precatalyst is also the off-loop resting state, in equilibrium with the active mononuclear form. Straightforward derivation of the rate law for this cycle yields a half-order in pre-catalyst. The experimental observation of half-order in pre-catalyst is generally an indication of radical mechanisms with reversible dimerization of the active catalyst. 


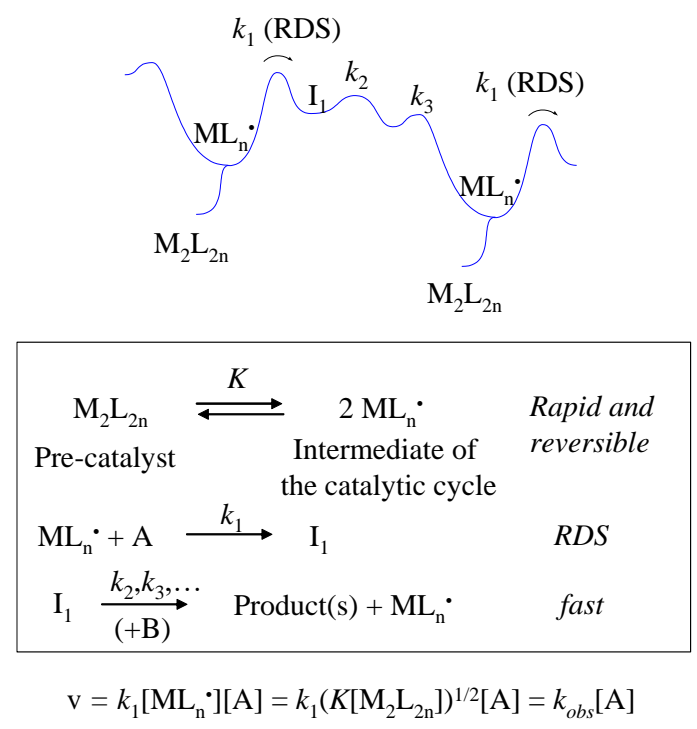

Figure 8. Example of a catalytic cycle showing a half-order in pre-catalyst.

The study of the reaction order in each of the substrates and other parameters (e.g. cocatalysts, mixed-solvent composition, $\mathrm{pH}, \ldots$ ) follows standard procedures, typically using large excess of all components except one, and verifying whether the transformation proceeds according to a pseudo-zero order or a pseudo-first order rate law. Analysis of the resulting $k_{o b s}$ as a function of the excess amount of the other components then allows derivation of the full rate law. Pre-equilibria and competitive forward and backward processes in high-energy intermediates generate complications in the rate law, with the observation of saturation behaviour, as they do for stoichiometric processes. Two limiting cases for the situation of a single intermediate between resting state and TS of the RDS are given in Figure 9, where the kinetic scheme and corresponding rate expressions are completely general (if a species indicated in parentheses is absent in the scheme, its concentration is replaced by 1 in the rate expression). More complex expressions result when more intermediates, each belonging to one of these two limiting situations, are involved. 


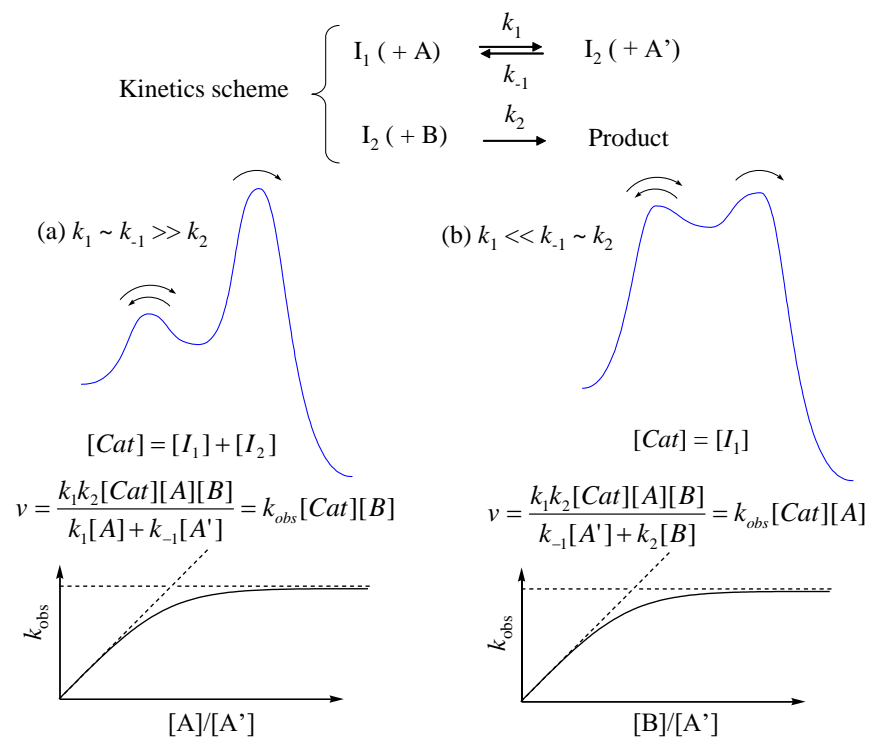

Figure 9. Rate laws for two limiting cases of the same single-intermediate kinetics scheme.

It is necessary to underline that knowledge of the full rate expression often requires extensive experimental studies with excursions into very wide concentration ranges, which may be difficult or impossible given the inherent limitations of the catalytic process. Thus, experimentally derived empirical rate expressions are sometimes given with fractional reaction orders, approximating the expected saturation trend in the intermediate concentration range used for their determination, and their validity is restricted within such range. An example is the expression $\mathrm{v}=k[\mathrm{Rh}][3,3-\mathrm{DMB}]^{0.1}\left[\mathrm{H}_{2}\right] /[\mathrm{CO}]$ derived for the $\mathrm{Rh}_{4}(\mathrm{CO})_{12}-$ catalyzed hydroformylation of 3,3-dimethylbutene. ${ }^{[8]}$ The small reaction order in olefin signals reversibility for the olefin coordination step, as well as for all subsequent steps (olefin insertion into the $\mathrm{Rh}-\mathrm{H}$ bond, $\mathrm{CO}$ coordination and $\mathrm{CO}$ insertion, see Figure 10) until the ratedetermining hydrogenolysis of the acyl intermediate, whereas the net inverse first order in $\mathrm{CO}$ is consistent with a dominant CO trapping relative to hydrogenolysis for the 16-electron acyl intermediate. $^{[9]}$ 


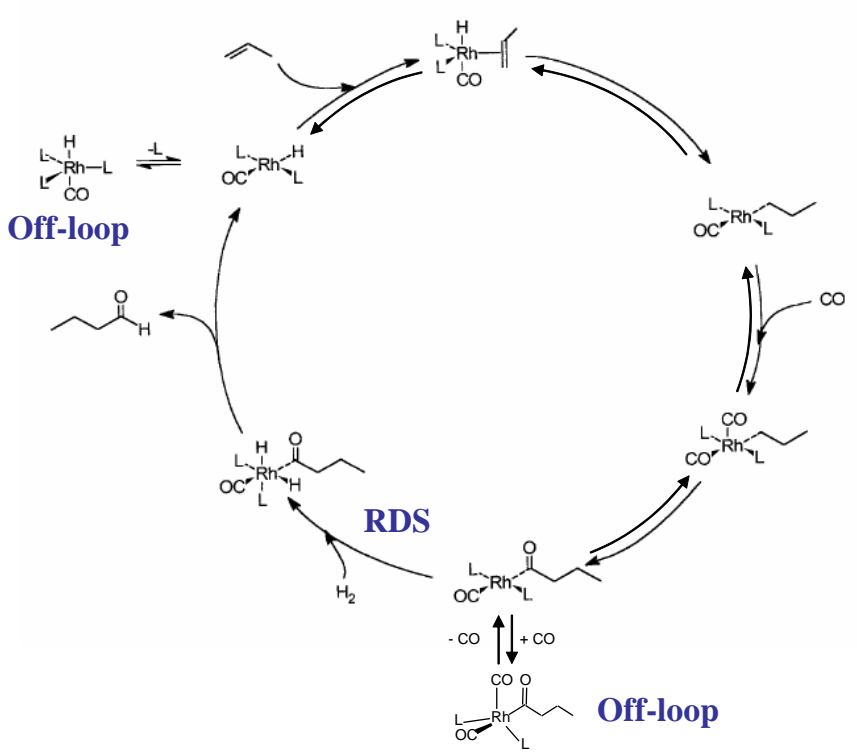

Figure 10. Proposed mechanism for olefin hydroformylation with $\mathrm{RhHL}_{3}(\mathrm{CO})$-type catalysts. $^{[9]}$

In summary, the kinetics investigation of a catalytic process provides useful information on the nature of the events and on the $G$ profile between the resting state and the transition state of the RDS. It also provides information on the activation parameters $\left(\Delta G_{\text {cycle; }}^{\ddagger}\right.$ also

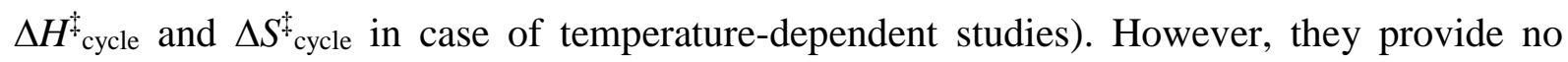
information on the faster steps of the catalytic cycle (after the RDS and until the next resting state). Furthermore, they provide no information whatsoever on the chemical identity of the catalytic intermediates.

\section{ISOTOPE LABELLING STUDIES}

The use of isotope labels provides information on the catalytic mechanisms essentially at three different levels: (a) they highlight differences in bonding patterns between the resting state and the TS of the RDS, through their effect on the rate constants; (b) they can distinguish between alternative mechanistic pathways through the distribution of the isotope labels in the 
products (regio-, stereoselecticity); (c) they provide information on the reversibility of certain elementary steps through the label incorporation into the unreacted substrate(s).

Most isotope labelling studies are carried out by using deuterium $\left({ }^{2} \mathrm{H}\right.$ or $\left.\mathrm{D}\right)$ in place of hydrogen $\left({ }^{1} \mathrm{H}\right)$, for several reasons: (a) reagents having D incorporated in specific positions are not too expensive or accessible by synthesis from inexpensive deuterated sources; $(b)$ the bonds experiencing change when going from the resting state to the TS of the RDS often contain $\mathrm{H} ;(c)$ the $\mathrm{H} / \mathrm{D}$ substitution provides the strongest isotope effect on the rate constant; (d) deuterium atoms are easily detected by NMR, either directly (even though D is a quadrupolar nucleus, the low quadrupole moment leads to sharp lines and reasonably high sensitivity) or indirectly in ${ }^{1} \mathrm{H},{ }^{13} \mathrm{C},{ }^{31} \mathrm{P}, \ldots$ NMR by observation of isotope shifts and scalar couplings $\left(\mathrm{J}_{\mathrm{HD}}, \mathrm{J}_{\mathrm{CD}}, \mathrm{J}_{\mathrm{PD}}, \ldots\right)$.

\subsection{Kinetic isotope effects}

The only relevant players to determine the extent of isotope effect on the turnover frequency are the catalyst resting state and the TS of the RDS, no matter how many elementary steps are in-between. As highlighted above (equation 3), the TOF is determined by $\Delta G^{\ddagger}$ cycle, but the isotopic substitution may only affect the energy component $(G=E+\mathrm{PV}-$ $\mathrm{TS}$ ), with no effect on the PV and $S$ terms. The effect on $E$ is expressed through the zeropoint vibrational energy (ZPVE). The isotopic substitution modifies the frequency of the normal modes of vibration, as given by $v=(\mathrm{k} / \mu)^{1 / 2}$, through its effect on the reduced mass $\mu$, whereas the force constant $\mathrm{k}$ is not affected. The zero-point energy (residual nuclear kinetic energy at $0 \mathrm{~K}$ ) is given by $1 / 2 \mathrm{~h} v$ under the harmonic approximation for each one of the $3 \mathrm{~N}-6$ normal modes. The reduced mass $\mu$ is dramatically affected if the normal mode of vibration has strong participation of a molecular motion involving bonds to H/D. The strongest expected effect is $\mu(D) / \mu(H)=2$ for an uncoupled H/D-X stretching vibration with a heavy $X$ 
atom or group $\left(m_{X} \gg m_{H}, m_{D}\right)$, giving rise to $v_{H X} / v_{D X}=(2)^{1 / 2}$. The ZPVE gain in this limiting case is therefore $\Delta\left(Z P E_{H / D}\right)=1 / 2 h\left[v_{H X}-v_{D X}\right]=1 / 2 h v_{H X}\left(1-2^{-1 / 2}\right)=0.146 \cdot h v_{H X}$. This difference is greater for higher vibrational frequencies, as schematically shown in Figure 11.

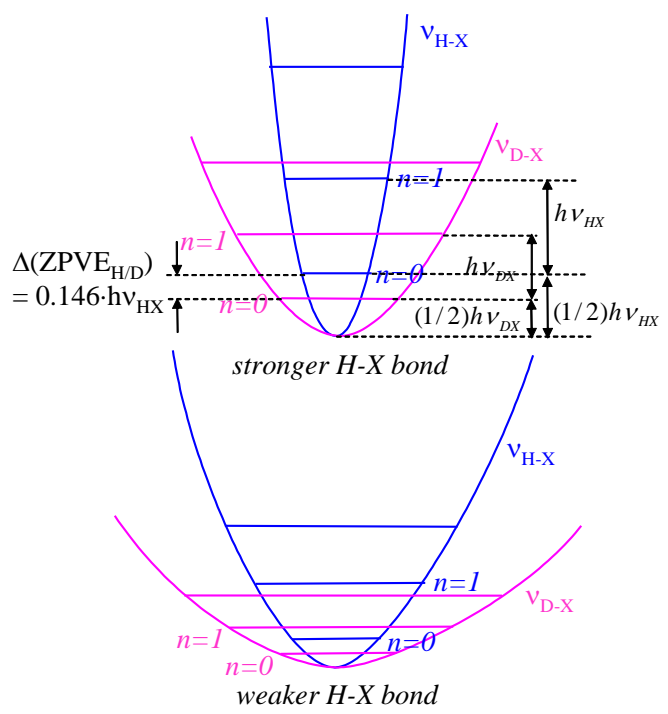

Figure 11. Effect of $H / D$ exchange on a normal mode of vibration with $\mu(D) / \mu(H)=2$.

For a situation in which a X-H bond of the resting state is partially or completely broken at the TS(RDS) level, substitution of $\mathrm{H}$ with $\mathrm{D}$ at this specific position leads to a greater stabilization of the resting state and a smaller (or zero) stabilization of the TS, thus increasing $\Delta \mathrm{G}_{\text {cycle }}^{\ddagger}$ and slowing down the catalytic process $\left(k_{\mathrm{H}} / k_{\mathrm{D}}>1\right)$. This is called a normal kinetic isotope effect (KIE), whereas the opposite situation (stronger $\mathrm{H}-\mathrm{X}$ bond in the TS relative to the resting state) leads to $k_{\mathrm{H}} / k_{\mathrm{D}}<1$ (inverse KIE) and is less frequent.

The numerical value of the KIE may vary in a very broad range depending on the number and strength of the bond(s) involved and on the temperature. The contribution of the $\mathrm{X}-\mathrm{H}$ stretching mode, in the limiting scenario where the bond is completely broken at the TS level, is given in Table 1 for a few $v_{\mathrm{X}-\mathrm{H}}$ and temperature values. The overall KIE value is obviously determined by the overall ZPVE change between resting state and TS(RDS), 
comprising all 3N-6 normal modes, resulting from all bond breaking and bond forming contributions. It may be affected by the solvent when this can interact with the molecules and change the vibrational structure at the resting state and/or TS(RDS) levels. A low value may result in certain cases, but any significant deviation from 1 provides quite important information, at least at the qualitative level, about changes at the given bond between the two kinetically relevant states. More quantitative information is often difficult to extract, except by comparison with high-level computations. In some cases higher KIE values relative to those shown in Table 1 may result, even up to 100! Such high values are indicative of tunnelling effects and, as such, they also help clarifying the shape of the PES in the proximity of the TS(RDS).

Table 1. Theoretical maximum contribution to $k_{\mathrm{H}} / k_{\mathrm{D}}$ (limiting case of full bond breaking at the TS level) by a single X-H/D stretching mode.

\begin{tabular}{|l|c|c|c|}
\hline$v / \mathrm{cm}^{-1}$ & \multicolumn{3}{|c|}{$k_{\mathrm{H}} / k_{\mathrm{D}}(\max )$} \\
\hline & $(\mathrm{T}=273 \mathrm{~K})$ & $(\mathrm{T}=298 \mathrm{~K})$ & $(\mathrm{T}=373 \mathrm{~K})$ \\
\hline 3600 & 15.6 & 12.4 & 7.5 \\
\hline 3400 & 13.4 & 10.8 & 6.7 \\
\hline 3100 & 10.7 & 8.7 & 5.7 \\
\hline 2800 & 8.5 & 7.1 & 4.8 \\
\hline
\end{tabular}

An example of the utility of KIE is given by the transfer hydrogenation of acetophenone (acp) with formic acid, catalyzed by the $\eta^{6}$-cymene complex (cymene) $\mathrm{RuCl}\{\mathrm{TsNCH}(\mathrm{Ph})-$ $\left.\mathrm{CH}(\mathrm{Ph}) \mathrm{NH}_{2}\right\}$. The proposed cycle is shown in Figure 12, where the boxed species have been isolated and characterized. The cycle and the presence of the indicated equilibria agree with the form of the rate law, but the details of the rate-determining transformation of the hydride intermediate (top of the cycle) to the diamido species (bottom of the cycle), in particular whether or not the hydridic and protic $\mathrm{H}$ atoms are transferred simultaneously, cannot be established by the rate law. Therefore, experiments with D labels were carried out. ${ }^{[10]} \mathrm{A}$ positive KIE was measured when using either $\mathrm{D}_{2} \mathrm{O}$ in place of $\mathrm{H}_{2} \mathrm{O}$ (the source of the protic $\mathrm{H}$ 
atom: $k_{\mathrm{RuHNH}} / k_{\mathrm{RuHND}}=1.68 \pm 0.10$ ) or $\mathrm{DCOO}^{-}$in place of $\mathrm{HCOO}^{-}$(the source of the hydridic $\mathrm{H}$ atom: $k_{\mathrm{RuHNH}} / k_{\mathrm{RuDNH}}=2.24 \pm 0.10$ ), and simultaneous use of both deuterated sources gave an even higher $\mathrm{KIE}\left(k_{\mathrm{RuHNH}} / k_{\mathrm{RuDND}}=3.05 \pm 0.50\right)$. On the other hand, the rate constant measured in DMF- $d_{7}$ is indistinguishable from that measured in regular DMF $\left(k_{\mathrm{DMF}} / k_{[\mathrm{D} 7] \mathrm{DMF}}=\right.$ 1.06 \pm 0.10 ). These experiments confirm that the bonds to both $\mathrm{H}$ atoms are significantly altered on going from the resting state to the TS(RDS) and suggest that the solvent does not play a significant role in this process.

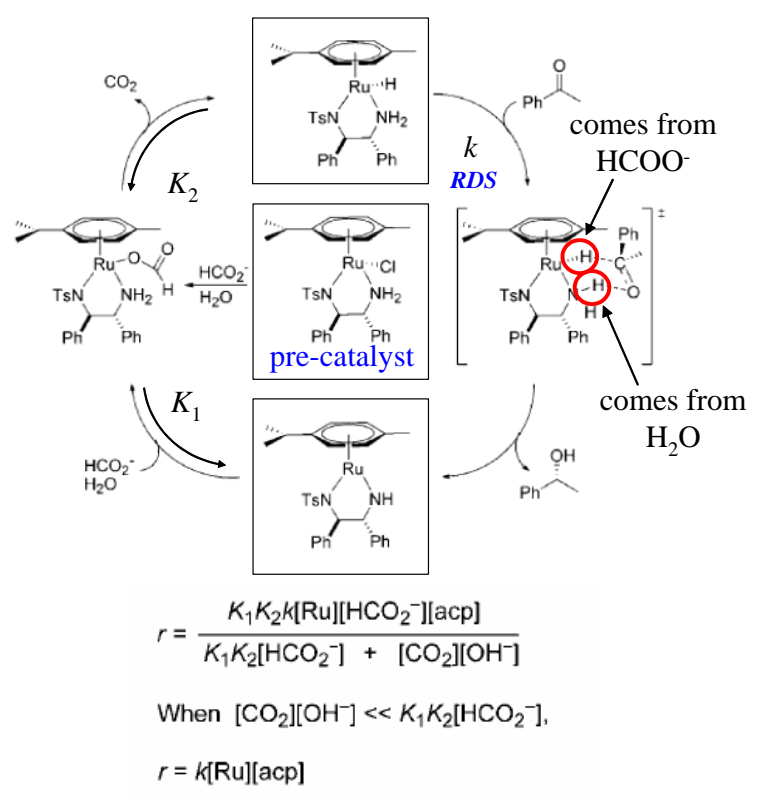

Figure 12. Proposed mechanism for the transfer hydrogenation of acetophenone (acp) with formic acid catalyzed by (cymene) $\mathrm{RuCl}\left\{\mathrm{TsNCH}(\mathrm{Ph}) \mathrm{CH}(\mathrm{Ph}) \mathrm{NH}_{2}\right\} .{ }^{[10]}$

Many other examples of the use of $\mathrm{D} / \mathrm{H}$ exchange for the determination of the KIE in catalysis can be found in the current literature. The use of KIE for the elucidation of catalytic mechanisms, however, is not limited to H/D. Important information has also been obtained by use of $k_{12} \mathrm{C} / k_{13 \mathrm{C}}$. This KIE is not obtained by measuring the rate in the presence and absence of labelled $\mathrm{C}$ atoms at specific positions, because this procedure would require rather expensive reagents and the sought information would suffer from low precision since the 
effect is expected to be quite small. A simple trick, which only requires the use of reagents at natural ${ }^{13} \mathrm{C}$ abundance, is to run the catalytic process until very high conversion and recover the unused substrate of interest. ${ }^{[11]}$ If a significant KIE arises from the ${ }^{13} \mathrm{C} /{ }^{12} \mathrm{C}$ substitution at any position, then the substrate will convert at significantly different rates, enriching the leftover substrate in one of the two isotopes at that particular position (e.g. the ${ }^{13} \mathrm{C}$ fraction will increase in the presence of a normal KIE). The unused substrate must be recovered in sufficiently large amounts to run a high resolution proton-coupled ${ }^{13} \mathrm{C}$ spectrum where the various $\mathrm{C}$ resonances can be quantified by integration with sufficiently high precision, with respect to an internal standard known (or assumed) to have no isotope effect. The needed level of substrate conversion depends on the extent of the KIE, because the proportion of a minor isotopic component $(R)$ in recovered material relative to the starting material $\left(R_{0}\right)$ is related to the fractional conversion $(F)$ and the KIE by equation 4. ${ }^{[11]}$

$$
R / R_{0}=(1-F)^{(1 / \mathrm{KIE})-1}
$$

An example of this practice is shown by the hydroamination of styrene with aniline catalyzed by the $\operatorname{Pd}(\mathrm{OTf})_{2} / \mathrm{dppf}^{[12]}$ The pre-catalyst is assumed to transform into the catalytically active diphosphine hydrido complex shown in Figure 13, which then coordinates and inserts styrene to yield a $\eta^{3}$ methylbenzyl derivative, the isolation and characterization of which suggests its role as catalytic resting state. ${ }^{[13]}$ Running the reaction up to $90 \%$ conversion and ${ }^{13} \mathrm{C}$ NMR analysis of the recovered styrene led to the calculation of the KIE values shown in Figure 13 for 4 independent experiments. In this study, the styrene para $\mathrm{C}$ atom (sufficiently far away from the reactive part of the substrate) was assumed to have no isotope effect. The absence of a significant deviation from unity for the meta, ortho and ipso $\mathrm{C}$ atoms comforts this assumption. The vinyl $\beta-\mathrm{C}$ atom also shows an insignificant $\mathrm{KIE}$, 
whereas the value determined for the $\alpha-C$ atom is significantly greater than 1 . This observation is consistent with the proposed catalytic cycle, where the $\operatorname{Pd}-(\alpha-C)$ bond is substantially weakened on going from the resting state to the TS of the rate-determining step.

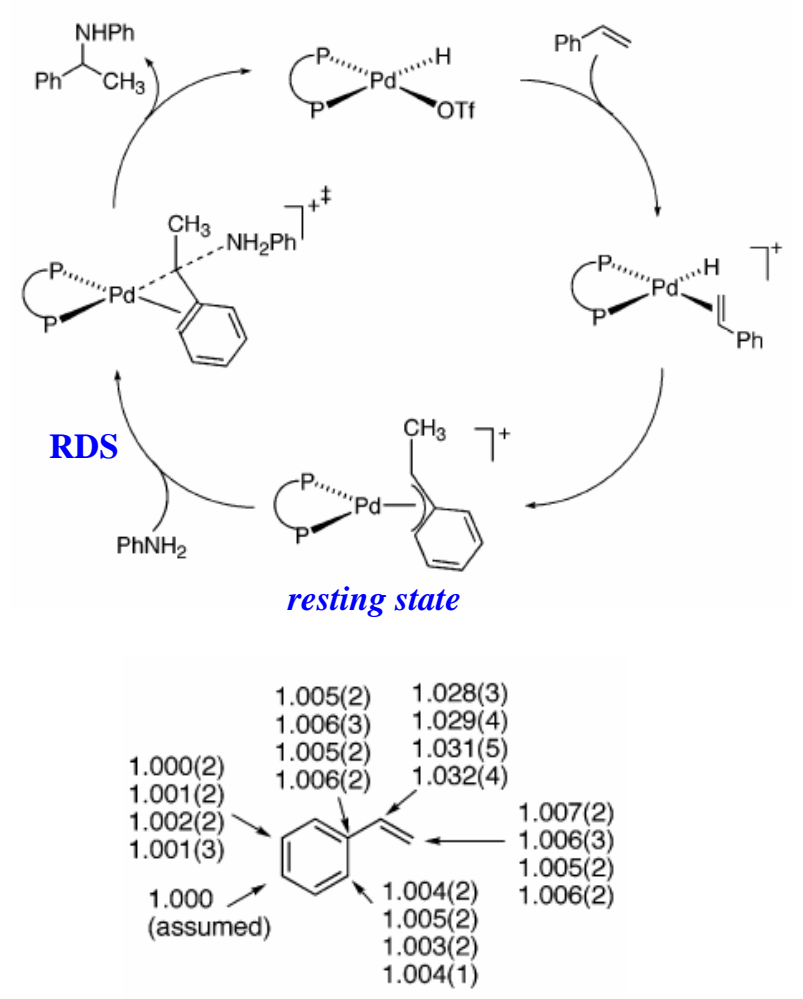

Figure 13. Proposed mechanism and $k_{12 \mathrm{C}} / k_{13 \mathrm{C}}$ results for the hydroamination of styrene with aniline catalyzed by $\mathrm{Pd}(\mathrm{OTf})_{2} / \mathrm{dppf}^{[12]}$

An alternative to the determination of small isotope effects in material labelled only at natural abundance is to employ the high precision of isotope ratio mass spectrometry, ${ }^{[14]}$ but a major restriction is that each site of interest must be selectively degradable, without affecting the isotope ratio, into an analyzable small molecule (for instance, $\mathrm{CO}_{2}$ for the measurement of the $\left.{ }^{13} \mathrm{C} \mathrm{KIE}\right)$. The advantage of the NMR technique is that it provides the simultaneous determination of all individual KIEs by direct measurement without further chemical transformation. 
Running title: Mechanisms in Homogeneous Catalysis

\subsection{Distribution of the isotope labels in the products}

The utility of this technique for the elucidation of catalytic mechanisms is obvious and is best illustrated with a couple of examples. As alluded to above, transfer hydrogenation of ketones may take place by a variety of mechanism. Four different ones have been proposed, as shown in Figure 14. They consist of the "Noyori-Morris" or "outer sphere" mechanism (A), two "inner-sphere" hydride mechanisms involving ketone coordination, insertion into the metal-hydride bond, and product elimination (distinguished as a "monohydride" (B) and a "dihydride" (C) version), and an "inner-sphere" direct transfer from the reducing agent to the coordinated substrate (D, a metal-templated, Meerwein-Ponndorf-Verley-type concerted mechanism). The KIE study discussed above (Figure 12) refers to a mechanism of type A.
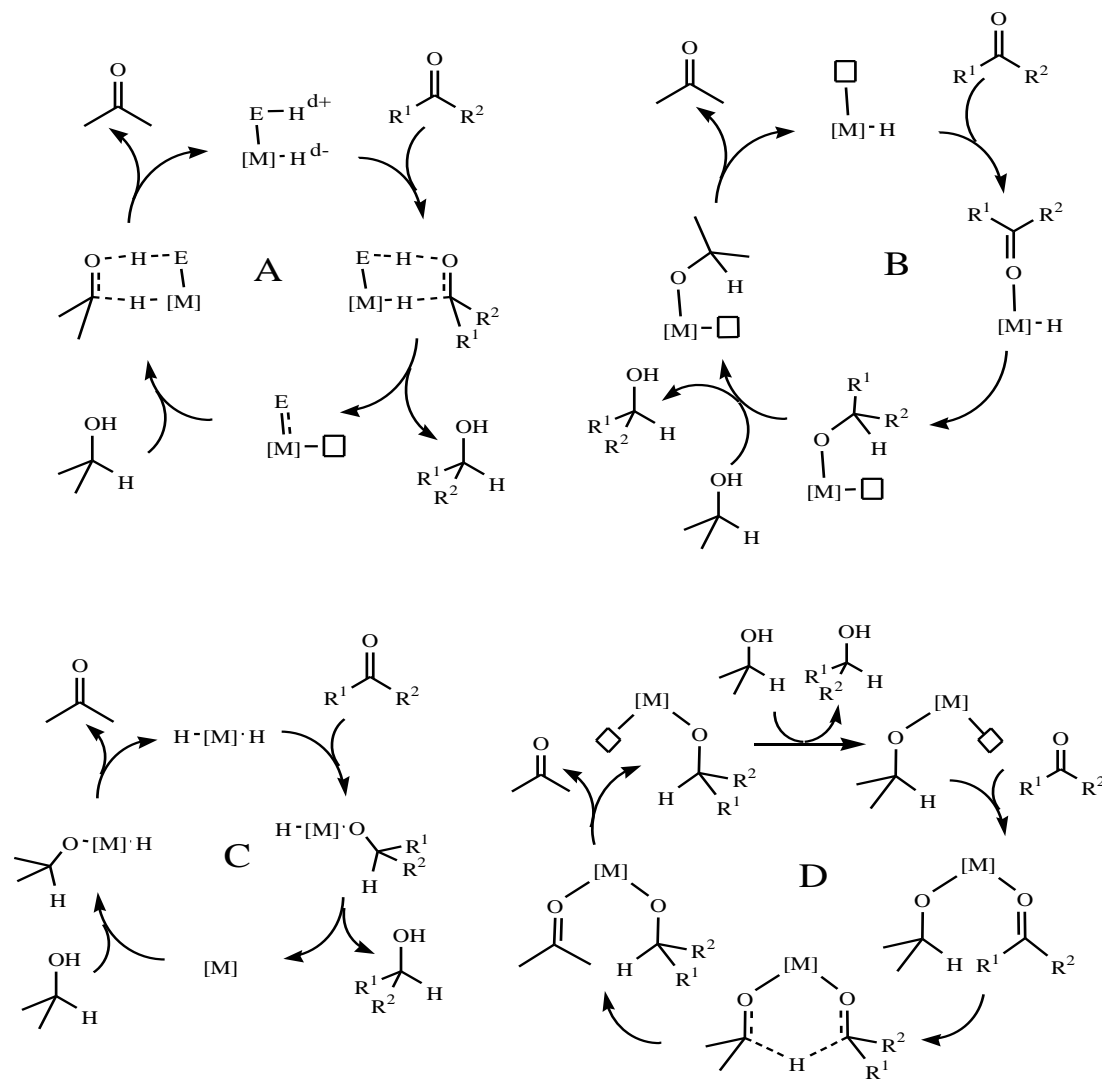

Figure 14. Mechanistic pathways for hydrogenation and transfer hydrogenation of ketones. 
The "dihydride" mechanism $\mathrm{C}$ is distinguished from the other by the fate of the two $\mathrm{H}$ atoms. For A, B and D, the "hydridic" $\mathrm{H}$ atom in the reductant (e.g. the $\alpha-\mathrm{H}$ atom of isopropanol) selectively becomes the "hydridic" atom in the product, whereas in cycle C this atom is distributed with equal probability to the $\mathrm{CH}$ and $\mathrm{OH}$ positions. Thus, use of selectively deuterated reductants such as $d_{1}$-cyclohexanol or racemization studies of an optically active $\alpha$-deuterated alcohol such as $d_{1}$-phenylethanol allow excluding at least one mechanistic possibility. Screening various catalysts led Pàmies and Bäckvall to the observation of nearly $100 \%$ of $\alpha-\mathrm{D}$ in the product in many cases, excluding mechanism C for these systems, but close to $50 \%$ for others, excluding mechanisms A, B and D for these. ${ }^{[15]}$ Note, however, that cycles A, B and D cannot be told apart by this experiment.

A second example is provided by olefin hydroamination, for which two alternative pathways, one involving olefin activation and the second one starting with activation of the amine (see Figure 15) are commonly considered. ${ }^{[16]}$ A particular system where either mechanism could in principle be operative is that based on the $\left[\mathrm{IrCl}(\text { olefin })_{2}\right]_{2} /$ phosphine precatalyst for the hydroamination of norbornene, originally developed by Casalnuovo et al. ${ }^{[17]}$ and later improved by Togni et al. ${ }^{[18]}$ Recent mechanistic investigations by Hartwig et al. using $\mathrm{PhND}_{2}$ demonstrate that the $\mathrm{D}$ label is selectively incorporated in a position syn with respect to the PhND sustituent, in agreement with the amine activation pathway. The olefin activation pathway seems excluded for this catalyst, because the amine attack anti relative to the metal would necessarily lead to the formation of the anti deuterated product. ${ }^{[19]}$ 

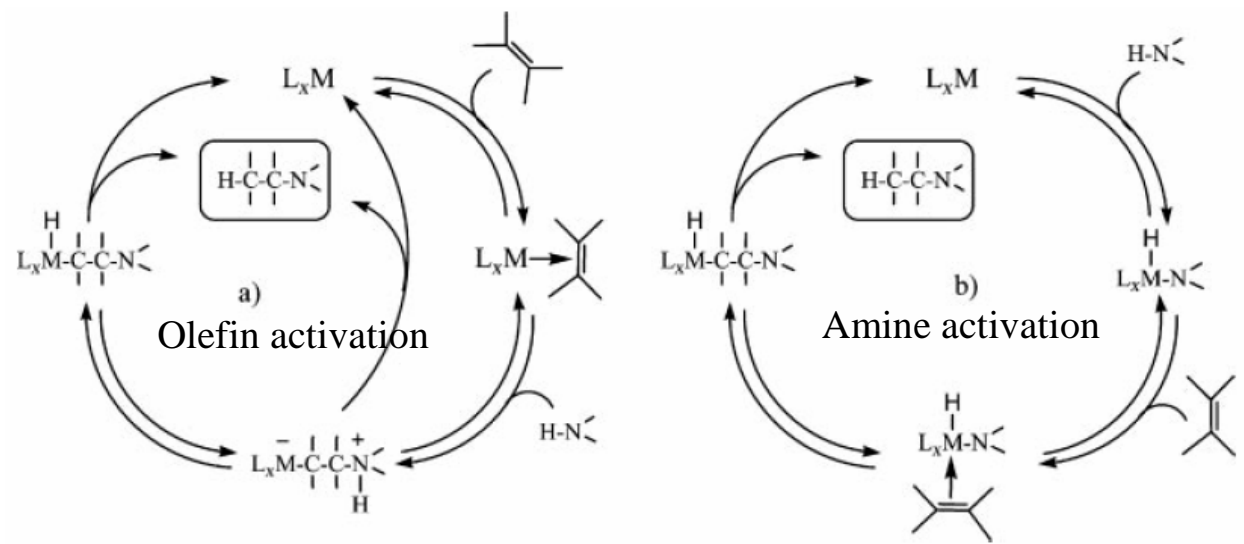

Figure 15. Two alternative pathways for intermolecular olefin hydroamination.

\subsection{Reversibility of substrate activation steps}

Incorporation of isotope labels is the best method to verify whether the substrate activation and a certain number of subsequent steps are reversible. The experiment requires stopping the catalytic transformation before complete conversion and analysing the isotope content in the unreacted substrate. Once again, the utility of this method is best illustrated by an example. In the olefin hydroformylation reaction, as already shown in Figure 10, the rate law suggests reversibility for a few of the initial steps. The hydroformylation of styrene with pre-catalysts of type $[\mathrm{RhCl}(\mathrm{COD})]_{2} /(\mathrm{P}, \mathrm{N})$, where $(\mathrm{P}, \mathrm{N})$ indicates a bifunctional phosphineamine ligand, yields a typical branched/linear ratio around 95:5 for the expected $\mathrm{PhCH}(\mathrm{CHO}) \mathrm{CH}_{3}$ and $\mathrm{PhCH}_{2} \mathrm{CH}_{2} \mathrm{CHO}$ product. The transformation proceeds in the presence of $\mathrm{D}_{2} \mathrm{O}$ to yield deuterated products, mainly $\mathrm{PhCH}(\mathrm{CHO}) \mathrm{CH}_{2} \mathrm{D}$, indicating exchange of the active $\mathrm{Rh}-\mathrm{H}$ intermediate with $\mathrm{D}_{2} \mathrm{O}$ to yield $\mathrm{Rh}-\mathrm{D}$, which then places the deuterium atom on the methyl group of the branched product upon 1,2-insertion of styrene, as shown in Figure 16. ${ }^{[20]}$ When the transformation was carried out up to $30 \%$ conversion, analysis of the recovered unreacted styrene revealed the presence of a significant amount of $\mathrm{PhCH}=\mathrm{CHD}$, but no $\mathrm{PhCD}=\mathrm{CH}_{2}$. As outlined in Figure 16, coordination of styrene and insertion into the Rh-D bond leads to either $\mathrm{Rh}-\mathrm{CH}_{2} \mathrm{CHDPh}$ (for 2,1 insertion, ultimately leading to the linear 
hydroformylation product) or $\mathrm{Rh}-\mathrm{CH}(\mathrm{Ph}) \mathrm{CH}_{2} \mathrm{D}$ (for 1,2 insertion, leading to the branched product). Reversal of both steps, with $\beta-\mathrm{H}$ elimination rather than $\beta$-D elimination, liberates $\mathrm{PhCD}=\mathrm{CH}_{2}$ and $\mathrm{PhCH}=\mathrm{CHD}$, respectively. Thus, in addition to demonstrating the reversibility of the styrene coordination step, the observation of the former and the absence of the latter confirms reversibility for 1,2 insertion, but not for 2,1 insertion. The reversibility of the first 2 steps in the branched cycle was also confirmed by the observation, at high conversion, of the doubly deuterated branched product, $\mathrm{PhCH}(\mathrm{CHO}) \mathrm{CHD}_{2}$.

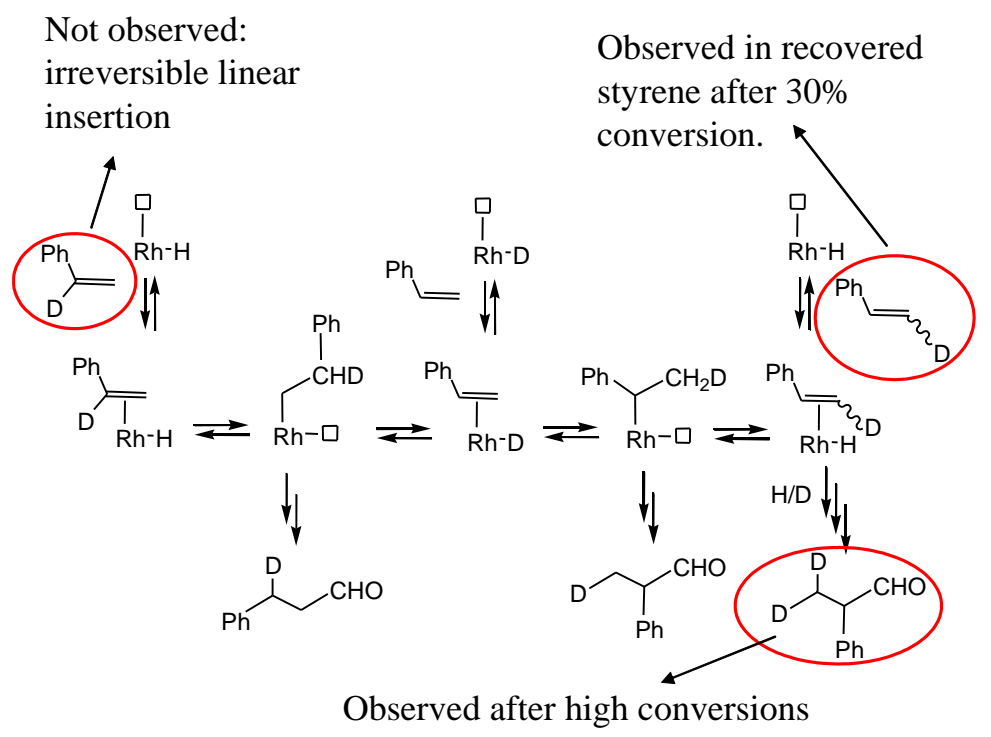

Figure 16. Pathways leading to $\mathrm{D}$ incorporation from $\mathrm{D}_{2} \mathrm{O}$ into unreacted substrate and products during Rh-catalyzed styrene hydroformylation.

A limitation of this method is that it provides only qualitative information. The extent of isotope incorporation into the unreacted starting material depends on several parameters, including the free energy difference between free substrate and the intermediate leading to isotope exchange, the relative activation barriers $\left(\Delta G^{*}\right)$ of the two processes that take this intermediate either back to free substrate or to final product, and the extent of substrate conversion. Thus, no quantitative information on the energy profile can be extracted from this analysis. 


\section{STOICHIOMETRIC STUDIES ON ISOLATED INTERMEDIATES}

In fortunate cases, catalytic intermediates can be isolated by running stoichiometric reactions between a suitable pre-catalyst and one or more of the reagents. This requires stability of the intermediate with respect to all possible irreversible deactivation processes under the conditions chosen for the synthetic and isolation procedure. The synthesis needs not be carried out under conditions identical to those used in catalysis, for instance conditions avoiding deactivation such as lower temperatures, provided that the pre-catalyst activation can still efficiently lead to the sought species. Once a product is isolated and characterized, its direct implication in the catalytic cycle must be proven by as many methods as possible before concluding that this is indeed a catalytic intermediate. These methods may include: $(a)$ use of the isolated species to initiate catalysis without induction time and with the same activity/selectivities (or better) shown by the previously used pre-catalyst; $(b)$ comparison of the spectroscopic properties with those obtained by in situ monitoring techniques, if available; (c) study of subsequent stoichiometric steps, ultimately leading to the same products of the catalytic process.

An example of the application of the above principle was already shown in Figure 7, where the bis-solvento complex $\left[\mathrm{Rh}(\mathrm{Et}-\mathrm{DuPHOS})(\mathrm{MeOH})_{2}\right] \mathrm{BF}_{4}$ was first generated by hydrogenation of the pre-catalyst in the absence of the unsaturated substrate, and then used as catalyst. A related $\left[\mathrm{Rh}(R, R\right.$-dipamp $\left.)(\mathrm{MeOH})_{2}\right] \mathrm{BF}_{4}$ (although not initially described as such) was isolated as a relatively stable yellow solid and then used in catalytic experiments. ${ }^{[21]}$ The subsequent step of the catalytic cycle (coordination of the unsaturated substrate, see Figure 17), led to the isolation of the corresponding intermediate when using another diphosphine ligand, dppe, through the reaction of $\left[\mathrm{Rh}(\mathrm{dppe})(\mathrm{MeOH})_{2}\right] \mathrm{BF}_{4}$ with $\mathrm{PhCH}=\mathrm{C}(\mathrm{COOMe})-$ 
$\left(\mathrm{NHCOCH}_{3}\right)$, commonly abbreviated as $\mathrm{MAC}$, in the absence of $\mathrm{H}_{2}$. The product $[\mathrm{Rh}(\mathrm{dppe})(\mathrm{MAC})] \mathrm{BF}_{4}$ was isolated and characterized by NMR and by an X-ray diffraction study. ${ }^{[22]}$ The follow-up stoichiometric reaction of this material with $\mathrm{H}_{2}$ yielded the expected hydrogenation product of MAC with regeneration of $\left[\mathrm{Rh}(\mathrm{dppe})(\mathrm{MeOH})_{2}\right]^{+}$.

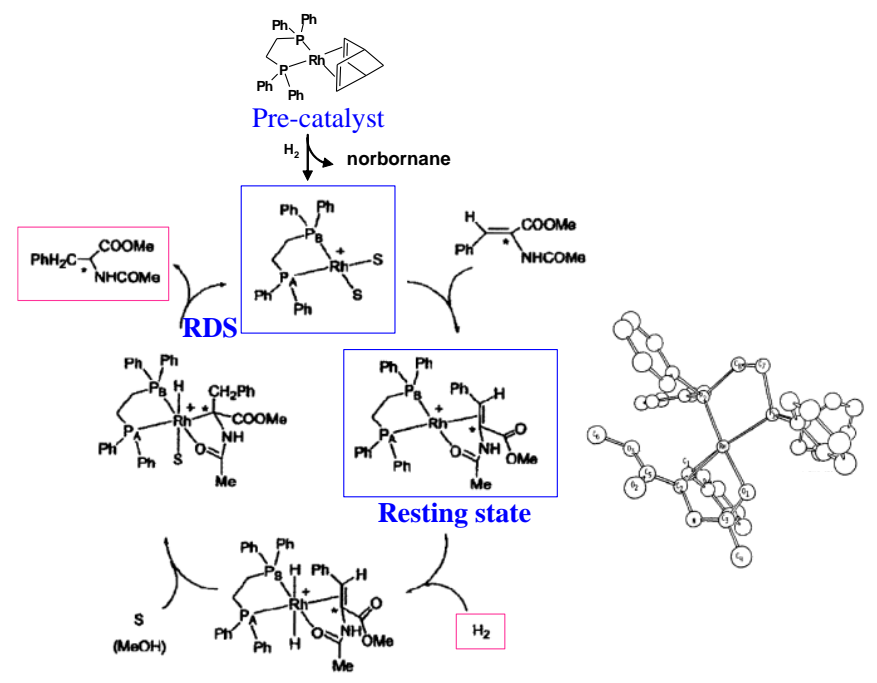

Figure 17. MAC hydrogenation catalyzed by $\left[\mathrm{Rh}(\mathrm{dppe})(\mathrm{MeOH})_{2}\right]^{+}$and structure of the $[\mathrm{Rh}(\mathrm{dppe})(\mathrm{MAC})]^{+}$intermediate.

Valuable information may also be furnished by studies on model compounds (complexes that are assumed to approximate the actual catalytic intermediates), that may be more accessible or easier to isolate or containing more suitable spectroscopic tags for reaction monitoring. These may be related to the real intermediates by: $(a)$ a change of metal (typically $5 \mathrm{~d}$ in place of $4 \mathrm{~d}$ since the former usually give slower transformations, thus kinetically more stable systems); (b) a change of neutral ligand (e.g. a carbonyl in place of a phosphine, or viceversa); (c) another ligand modifications (e.g. $\mathrm{Cl}$ in place of $\mathrm{H}$, Tp in place of $\mathrm{Cp}$, etc.). However, one must be extremely careful when interpreting the results of stoichiometric reactions obtained with model compounds because catalytic cycles often change by slight variations of substrates, ligand substituents, and reaction conditions, let alone deeper changes 
Running title: Mechanisms in Homogeneous Catalysis

as those mentioned above. At best, such studies provide useful hints about the chemical reactivity (stereochemistry, selectivities, ...) of the actual catalytic intermediates.

\section{IN SITU SPECTROSCOPIC INVESTIGATIONS}

These studies can be grossly divided into two classes, with two different objectives. The first class consists in the investigations under truly catalytic conditions, providing information on the nature of the resting state and other low-energy species of the cycle. In order to be really relevant, these studies must be carried out under the actual conditions (nature of the compounds, solvent, temperature and concentrations). The second class includes studies aimed at identifying and obtaining structural information on a specific intermediate. These studies are often carried out under any condition that optimizes the observation of the sought species. ${ }^{[5]}$

\subsection{In situ investigations of a catalytic run}

Carrying out an in situ spectroscopic study under conditions identical to the catalytic run is not always possible, for instance when the temperature is outside the range tolerated by the suitable monitoring instrumentation. Another common problem is that of the low catalyst concentrations (often $<1 \mathrm{mM}$ ), too low for detection with a reasonable signal-to-noise ratio by certain spectroscopic techniques. In those cases, experiments may be carried out at higher catalyst concentration, perhaps using lower temperatures or less active forms in order to slow down the reaction. However, metal-ligand equilibria may shift considerably upon concentrating the solutions, which hampers direct comparison of the data observed in catalysis and in situ monitoring. Thus, care must be exercised for extrapolation of the results in those cases. 
It is important to verify that the observed species is indeed catalytically relevant. As shown in Figure 3, many species may be simultaneously present including pre-catalyst, catalytic intermediates, off-loop species and deactivated forms. The spectroscopic signatures of the pre-catalyst are known, thus such species can be easily recognized. In addition, signals of pre-catalysts are expected to decrease continuously during the induction time. Conversely, signals of deactivated species increase with time in a way that can be correlated with the decrease of the catalytic activity. In an in situ study of a catalytic run, the concentration of a kinetically stable intermediate or off-loop species remain constant, or vary as a function of the reactants conversion in a way that can be related to the chemical equilibria in which the species is involved. In the latter case, the observed concentration changes provide useful information on the equilibria within the catalytic cycle.

Under the assumption that deactivated and not-yet-activated forms are absent, namely that only catalytic intermediates and off-loop species are present, the distribution of the observed species will reflect the relative energetics ( $\Delta G$ relative to the resting state) according to Boltzmann population statistics, all species being considered within the same cycle between two consecutive TS of the RDS (for instance, between two consecutive TS4 in the energetic profile of Figure 5). If all these species are mononuclear (thus their relative proportion is concentration independent) and considering the ideal case where all other species participating in the equilibria are present at $1 \mathrm{M}$ concentration, then $K_{\mathrm{x}}=\left[\mathrm{I}_{\mathrm{x}}\right] /[\mathrm{RS}]=$ $\exp \left(-\Delta G_{\mathrm{X}} / \mathrm{RT}\right)$, where $\mathrm{I}_{\mathrm{x}}=$ intermediate or off-loop species under consideration. Simple calculations show that this concentration ratio quickly becomes very small as $\Delta G$ increases, for instance $0.025,1.0 \cdot 10^{-4}$ and $1.0 \cdot 10^{-8}$ for $\Delta G=2,5$ and $10 \mathrm{kcal} \mathrm{mol}^{-1}$ at $273 \mathrm{~K}(0.068$, $1.2 \cdot 10^{-3}$ and $1.4 \cdot 10^{-6}$ for the same $\Delta G$ at $373 \mathrm{~K}$ ). This means that higher energy intermediates are only a tiny fraction of the total catalyst amount, which is itself already quite small. Only very sensitive techniques may detect higher energy intermediates in situ. The most readily 
detected species are only the resting state and other intermediates with a free energy very close to it.

Any analytical technique may be used in principle for in situ characterization, provided that: (a) it can be implemented under the same conditions of the catalytic runs (or close to); (b) it is sufficiently sensitive to detect the catalytic intermediates; $(c)$ it provides useful information to probe the chemical nature of the detected species. A non exhaustive list of the techniques that have often been applied to in situ study of catalytic cycles includes multinuclear NMR spectrometry (mostly ${ }^{1} \mathrm{H}$ for sensitivity reasons; structural information through chemical shifts, coupling constants, relaxation time data, diffusion constants, etc.), IR and Raman spectroscopy (absorption frequency, relative line intensity, linewidths, symmetry), UV/visible spectroscopy (absorption frequency, intensity, linewidths), mass spectrometry (m/z, distribution, e.g. in polymerization catalysis), EPR spectrometry (limited to paramagnetic species: $\mathrm{g}$, hyperfine splittings, zero-field splitting).

A special word needs to be spared about reactions involving high-pressure gases $\left(\mathrm{H}_{2}\right.$ in hydrogenation or hydroformylation, $\mathrm{CO}$ in carbonylations, $\mathrm{C}_{2} \mathrm{H}_{4}$ and other light olefins in polymerization, etc.). In situ studies of these reactions require the use of special high-pressure cells and indeed, such devices have been developed for spectroscopic techniques such as IR and NMR. ${ }^{[23]}$ For analytical techniques where high-pressure cells are not available, or when the operating pressures are too high for the available technology, monitoring may be carried out under less severe conditions, although once again the value of the resulting information may be questionable. A major problem with the use of static high-pressure cells is slow diffusion, causing shortage of the gaseous reactants and concomitant change in the equilibria involving these species. The consequence is that wrong information may be obtained concerning the nature of the resting state and the relative distribution of other low-energy 
species. To alleviate this problem, special flow-cells have been developed, allowing a continuous supply of gases and optimal mixing.

Another special word must be spared on the use of parahydrogen in ${ }^{1} \mathrm{H}$ NMR spectrometry. ${ }^{[24]}$ This technique, generally referred to as parahydrogen induced polarization (PHIP) NMR, is limited to the study of catalytic processes where $\mathrm{H}_{2}$ is consumed, but allows unparalleled sensitivity for the detection of low concentration catalytic intermediates, mostly hydride complexes. Parahydrogen is one of the two possible forms of $\mathrm{H}_{2}$, where the two nuclear spins have an antiparallel correlation. Since its energy is slightly lower than that of the other form (orthohydrogen), para-enriched $\mathrm{H}_{2}$ can be obtained by equilibration at very low $\mathrm{T}$ (typically liquid $\mathrm{N}_{2}$ is used, yielding a ca. 50:50 ortho/para distribution, vs. the 75:25 statistical distribution at high temperatures). The equilibration is extremely slow because symmetry forbidden, but is greatly accelerated by certain catalysts such as rust. After generation, para-enriched $\mathrm{H}_{2}$ can be kept essentially indefinitely. The utility of this reagent is that the nuclear spin correlation is maintained when the two $\mathrm{H}$ atoms become incorporated into a product, although the loss of symmetry speeds up the eventual loss of coherence. The consequence, when the two $\mathrm{H}$ atom are inequivalent in the product, is that among the four possible nuclear spin combinations $\alpha \alpha, \beta \beta, \alpha \beta$ and $\beta \alpha$, the last two are much more populated and the first two much less relative to the Boltzmann distribution, thus the four related resonances (two doublets for a coupled $\mathrm{H}_{\mathrm{A}} \mathrm{H}_{\mathrm{B}}$ system) are greatly enhanced, theoretically by a factor up to $>10^{4}$, and are observed as two enhanced absorptions and two enhanced emissions, looking as "antiphase" doublets. More complex patterns are seen in the presence of additional coupling (e.g. to the $\mathrm{P}$ nucleus of phosphine ligands or to a metal such as Rh). Such dramatic signal enhancements can also be transferred to lower sensitivity nuclei by polarization transfer methods, but application to low concentration catalytic species have so far mostly involved the measurement of ${ }^{1} \mathrm{H}$ signals. 
Application of this method to the olefin hydrogenation catalyzed by $\mathrm{RhCl}\left(\mathrm{PPh}_{3}\right)_{3}$ (Wilkinson's catalyst) has revealed new features, including the direct detection of the off-loop $\mathrm{RhH}_{2} \mathrm{Cl}\left(\mathrm{PPh}_{3}\right)_{3}$ complex and the $\mathrm{RhH}_{2} \mathrm{Cl}\left(\mathrm{PPh}_{3}\right)_{2}$ (olefin) intermediate (see Figure 18), as well as the identification of a second, slower hydrogenation cycle that occurs on dinuclear Rh complexes, the importance of which decreases in the presence of excess $\mathrm{PPh}_{3}{ }^{[25]}$

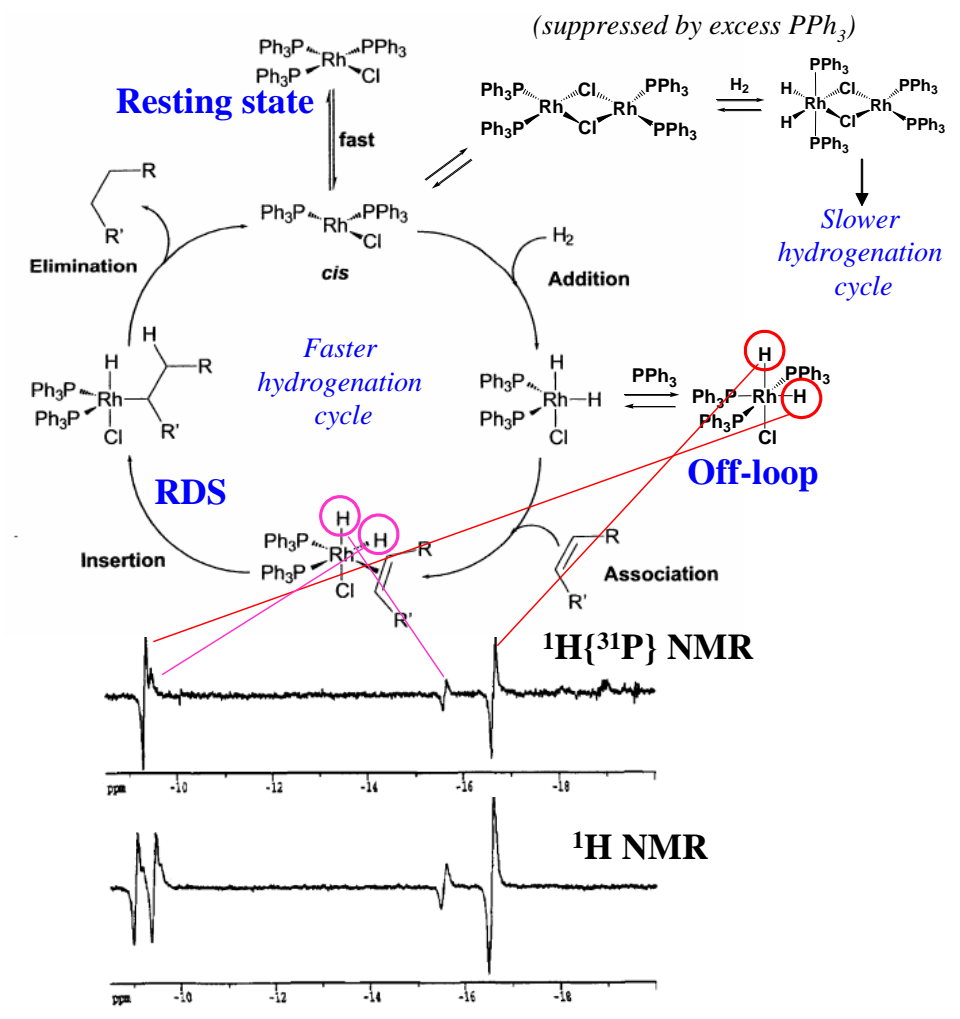

Figure 18. The olefin hydrogenation catalyzed by Wilkinson's catalyst reinvestigated by in situ ${ }^{1} \mathrm{H}$ PHIP NMR.

Another example of the application of an in situ spectroscopic study concerns the highpressure IR monitoring of olefin hydroformylation, which follows the general cycle already outlined in Figure 10. The activation process starting from the $\mathrm{Rh}(\mathrm{acac})(\mathrm{CO})_{2}$ pre-catalyst in the presence of a triaryl phosphite ligand $\mathrm{L}$ (Figure 19) generates compound $\mathrm{RhH}(\mathrm{CO})_{3} \mathrm{~L}$, characterized by bands at 2093, 2043 and $2013 \mathrm{~cm}^{-1}$, and an unknown species with bands at 2064 and $2031 \mathrm{~cm}^{-1} \cdot{ }^{[26]}$ After introduction of the olefin, the aldehyde product quickly grows, 
obscuring any carbonyl band of possible acyl intermediates in the IR spectrum. However, the use of a rapid scanning technique with monitoring during the very first moments after the olefin addition provides useful information. The results obtained for 1-octene are shown in Figure 19. The difference spectra illustrate the decrease of both $\mathrm{RhH}(\mathrm{CO})_{3} \mathrm{~L}$ and the unknown species, while a new compound characterized by bands at 2080, 2019, 1996 and $1690 \mathrm{~cm}^{-1}$ grows. By comparison with reference compounds, this spectrum can be assigned to the acyl derivative $\mathrm{Rh}(\mathrm{CO})_{3}(\mathrm{~L})\left(\mathrm{COC}_{8} \mathrm{H}_{17}\right)$. Running the same experiment with difference substrates shows similar results for all terminal olefins (1-octene, styrene and pentafluorostyrene), consistent with the resting state being the acyl species, whereas internal olefins such as cyclohexene do not yield any spectral change, indicating that the resting state in those cases is the hydride complex $\mathrm{RhH}(\mathrm{CO})_{3} \mathrm{~L}$. These results agree with indications independently obtained from the kinetics studies.

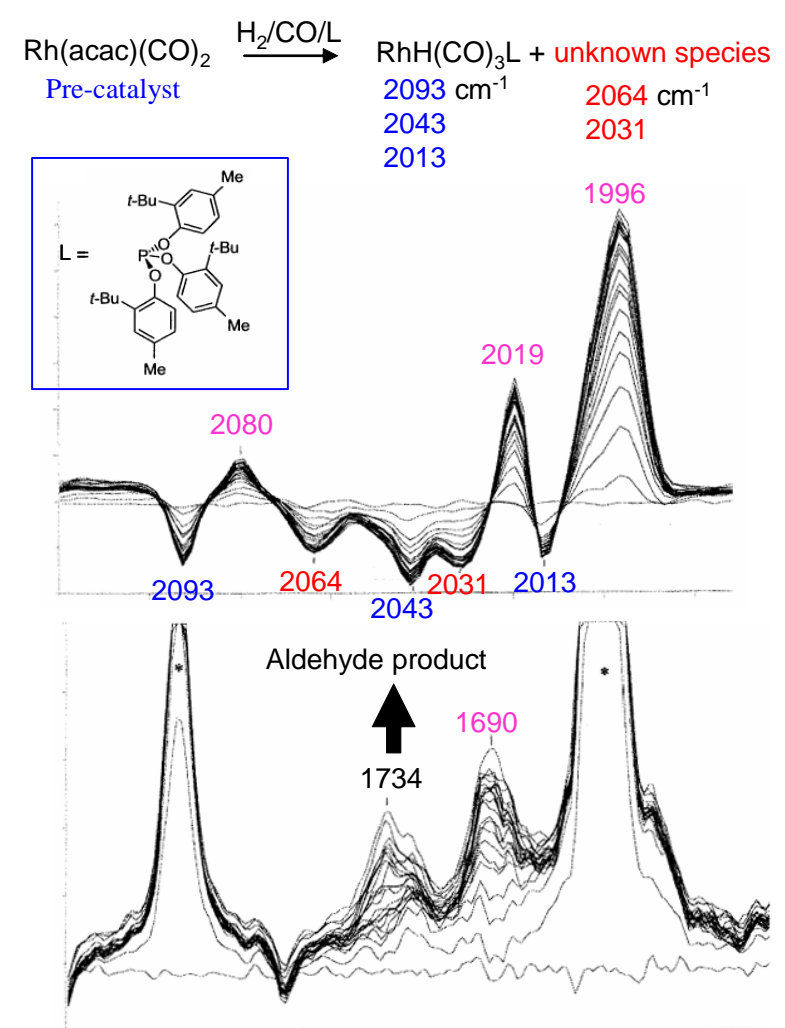


Figure 19. A high-pressure IR study of the 1-octene hydroformylation of olefin catalyzed by $\mathrm{Rh}(\mathrm{acac})(\mathrm{CO})_{2}$. The figure shows the difference spectra obtained after the addition of 1 -octene, subsequent to pre-catalyst activation by $\mathrm{H}_{2} / \mathrm{CO} / \mathrm{L}$. Spectra were collected at a rate of 80 spectra per second for a time span of $0.82 \mathrm{~s}$.

\subsection{Investigating the identity of missing intermediates}

Because of the concentration limitations described above, certain catalytic intermediates may never be detectable under truly catalytic conditions. Thus, additional information may be obtained by monitoring specific stoichiometric steps of the catalytic cycle. These studies may be carried out at higher concentrations of the metal species and in the presence of only those reagents implicated in the given step, but the purpose is not observing and possibly isolating the final product of the stoichiometric transformation (this topic was covered in a previous section), but rather to spectroscopically detect an intermediate and to elucidate its structural details. Demonstration that the observed stoichiometric transformation is indeed part of the catalytic cycle requires independent verification that the starting and ending compounds are themselves active catalysts for the process under consideration, with the same activity and selectivities under the same conditions. Thus, only isolated intermediates or off-loop species may be used as starting point. A pre-catalyst needing activation won't do. Typically, the resting state of the cycle is the compound most easily isolated and therefore most frequently used in this approach, but starting from a higher energy catalytic intermediate, if available, provides additional advantages by allowing other intermediates, at yet higher energy, to accumulate to higher concentrations becoming more easily observable. This detective task may also be facilitated by working under different reaction conditions, e.g. at very low temperature or in a different solvent, possibly resulting in a favourable change of the PES in favour of the sought intermediate, although once again care is needed for extrapolation of the results to the catalytic conditions. 
One example where this strategy was applied is related once again to the hydrogenation of unsaturated compounds catalyzed by $\left[\mathrm{Rh}(\mathrm{PP})(\mathrm{MeOH})_{2}\right]^{+}$-type complexes, with the mechanism already outlined in Figure 17. Treatment of the isolated resting state (the enamide complex $[\mathrm{Rh}(\text { dppe })(\mathrm{MAC})]^{+}$) with $\mathrm{H}_{2}$ in $\mathrm{CD}_{3} \mathrm{OD}$ at $-78^{\circ} \mathrm{C}$ for $3 \mathrm{~h}$ allowed the accumulation in solution and spectroscopic identification by ${ }^{1} \mathrm{H}$ and ${ }^{13} \mathrm{C}$ NMR of the alkyl-hydride intermediate that precedes the RDS. ${ }^{[27]}$ Success of this strategy in this particular case is related to the concurrence of several positive effects of the low temperature: $(a)$ the $\mathrm{H}_{2}$ solubility in methanol increases; $(b)$ the relative free energy shifts in favour of the alkylhydride intermediate because of the negative entropy change; $(c)$ the rate of the subsequent reductive elimination step decreases. Proof that the observed product is indeed a catalytic intermediate is readily provided by the observed release of the hydrogenated substrate and concomitant regeneration of $\left[\mathrm{Rh}(\mathrm{dppe})(\mathrm{MeOH})_{2}\right]^{+}$when the solution is warmed up.

Still remaining focused on this catalytic mechanism, the identity of the last missing intermediate, the olefin-dihydride compound, could be investigated by using a system with a different diphosphine ligand, PHANEPHOS, see Figure 20. ${ }^{[28]}$ The chosen conditions modulate the PES in favour of such intermediate and detection was facilitated by the signal enhancement provided by parahydrogen (vide supra). The assignment of the two observed hydride resonances to the dihydride species, rather than the alkyl-hydride product of subsequent olefin insertion, was supported by the strong coupling that both hydrides show with the $\mathrm{Rh}$ and $\mathrm{P}$ nuclei, as well as with each other. In addition, the signal around $\delta-2$ is further split by strong coupling when $100 \%{ }^{13} \mathrm{C}$-enriched MAC at the $\beta$ position was used. This suggests that the compound has in fact already established an interaction between the hydride and the coordinated olefin, with rapid equilibrium between the dihydride form and the hydride-alkyl form, or perhaps the insertion product keeps a strong agostic interaction that simultaneously allows strong H-Rh and H-P coupling. 


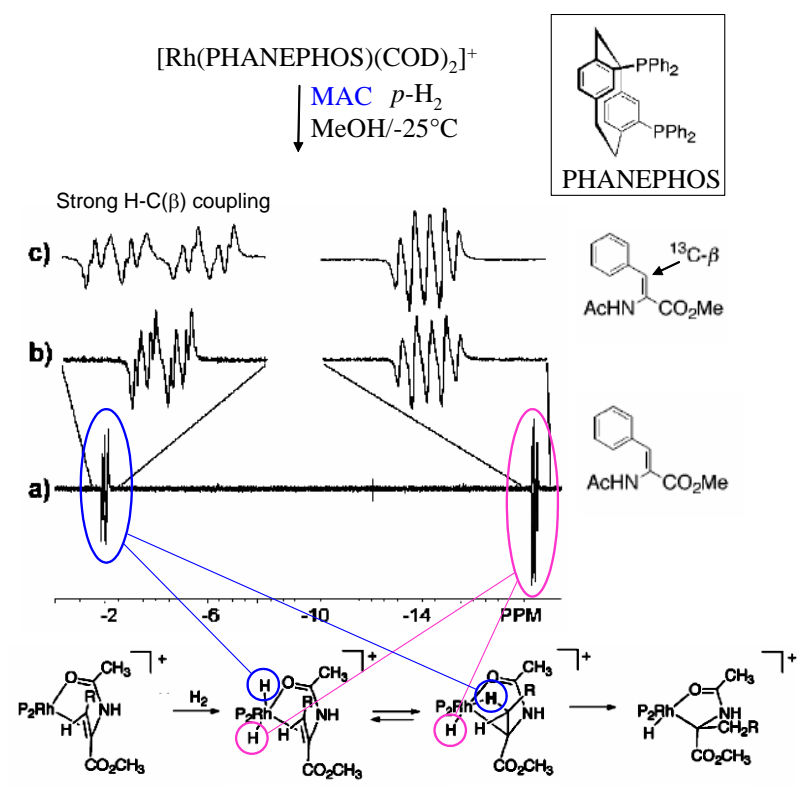

Figure 20. A ${ }^{1} \mathrm{H}$ PHIP NMR study of the MAC hydrogenation catalyzed by $\left[\mathrm{Rh}(\mathrm{PHANEPHOS})(\mathrm{MeOH})_{2}\right]^{+}$. The reaction was carried out in the presence of parahydrogen at 1 bar in $\mathrm{CD}_{3} \mathrm{OD}$ at $-25^{\circ} \mathrm{C}$.

\section{EFFECTS OF THE LIGAND STRUCTURE}

A last experimental strategy for the investigation of catalytic mechanisms consists of the examination of ligand effects. Ligand modification is at the very heart of homogeneous catalysis, with the obvious goals of maximizing the catalytic activity and the selectivity (chemo-, regio-, stereo-, enantio-) in favour of the desired reaction product. In turn, the analysis of how the measurable physical parameters ( $\Delta G^{\ddagger}$ cycle, selectivities) are affected by the ligand modification provides valuable information on the catalytic cycle, notably on the resting state and on the transition state of the RDS. Many studies of this type are available in the literature, because checking how the activity and selectivity of a catalytic reaction depend on the ligand substitution is not only systematically done for catalyst optimization, but it is also much simpler to execute experimentally than an in situ spectroscopic study or a complete kinetics investigation. As an illustrative example, Table 2 shows the results for the 
hydroformylation of 1-octene using a variety of THIXANTPHOS-type ligands. ${ }^{[29]}$ Electron donating substituents decrease the reaction rate, while the most active systems contain electron withdrawing groups $\left(\mathrm{CF}_{3}\right)$. This has been considered consistent with weakening of $\mathrm{CO}$ coordination to the acyl intermediate, speeding up the catalysis by raising the energy of the acyl resting state (see cycle in Figure 10). The 1:b ratio was found to correlate with the ratio of equatorial-equatorial (ee) and equatorial-apical (ea) isomers for the $\mathrm{Rh}$ (THIXANTPHOS)H(CO) 2 complex: the eelea ratio shifts gradually from approximately 1 for the $p-\mathrm{NMe}_{2}$ substituted ligand to more than 9 for the $p-\mathrm{CF}_{3}$ substituted ligand.

Table 2. Activity and selectivities observed in 1-octene hydroformylation catalyzed by $\mathrm{Rh}$ complexes containing THIXANTPHOS-type bidentate ligands. ${ }^{[29]}$

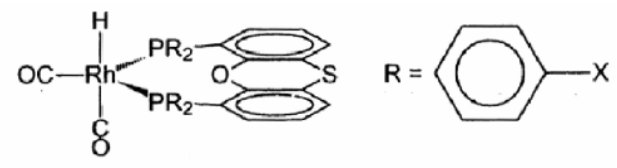

\begin{tabular}{lccc}
\hline $\mathrm{X}$ & Rate $^{\mathrm{a}}$ & \% $^{\text {Isomer. }}{ }^{\mathrm{b}}$ & $\mathrm{l:b}^{\mathrm{c}}$ \\
\hline $\mathrm{CF}_{3}$ & 158 & 7 & 89 \\
$\mathrm{Cl}$ & 68 & 7 & 68 \\
$\mathrm{H}$ & 107 & 5 & 50 \\
$\mathrm{~F}$ & 75 & 6 & 52 \\
$\mathrm{Me}$ & 78 & 5 & 44 \\
$\mathrm{OMe}$ & 45 & 6 & 37 \\
$\mathrm{NMe}_{2}$ & 29 & 5 & 45 \\
\hline
\end{tabular}

${ }^{\text {a }}$ Rate in $\mathrm{mol} \mathrm{h}^{-1} .{ }^{\mathrm{b}} \%$ isomer $=$ selectivity for 2 -octene. ${ }^{\mathrm{c}} \mathrm{l} \cdot \mathrm{b}=$ linear to branched ratio of aldehyde product.

Another way in which ligand variation can help for the elucidation of catalytic mechanisms is by assisting the in situ spectroscopic investigation of catalytic intermediates, through suitable modification of the PES, thus raising the relative concentration of a particular intermediate, and/or by introducing more easily interpretable spectroscopic tags. Examples of these approaches have already been given in the previous sections.

\section{THEORETICAL CALCULATIONS}


Given the spectacular recent progress of computational speed and the availability of user-friendly software packages for electronic structure calculations, the application of computational tools to the elucidation of stoichiometric and catalytic mechanisms has become commonplace. ${ }^{[4,6]}$ In addition, while in the past the computational approach was reserved to expert theoretical chemists, it has now become possible for experimentalists who possess a good understanding of the basic quantum chemistry principles to use themselves this methodology and the number of original research papers published by experimentalists that include a computational study is constantly growing. In the old days computational tools could only be applied to the electronic structure calculation of fixed geometries, mainly by semiempirical methods, and typically on simplified structural models (e.g. by replacing most substituents directly linked to donor atoms with $\mathrm{H}$, e.g. using $\mathrm{PH}_{3}$ in place of trialkyl or triaryl phosphines), in order to understand specific features of the chemical bonding, or to assess the likelihood of a particular structure to be "stable". Nowadays, molecular geometries can be optimized to match very closely the experimentally available (e.g. by X-ray diffraction) structural data and relative energies can be obtained with relatively good precision (within 2-5 $\mathrm{kcal} \mathrm{mol}^{-1}$ from the experimental data, depending on the computational accuracy), for molecules containing tens of atoms and hundreds of electrons. Thus, it has become possible to use computations to explore the details of a catalytic pathway.

Theoretical calculations can provide useful information by optimizing the geometry and providing the relative energy of all stationary points along the reaction coordinate. These are all the structures where the energy gradient is zero along all $3 \mathrm{~N}-6$ internal coordinates and are classified as (a) local minima (positive curvature along all 3N-6 internal coordinates: catalytic intermediates and off-loop species) and (b) saddle points (negative curvature along one coordinate - the reaction coordinate - and positive curvature along all other $3 \mathrm{~N}-7$ coordinates: 
transition states). A particular situation occurs when the mechanism involves a change of spin (crossing from one PES of spin S to another surface, usually differing in spin by one unit, $\mathrm{S} \pm 1) .{ }^{[30]}$ In those cases the crossing point may constitute a critical point of the energetic profile (e.g. a transition state) and software permitting its geometry optimization and the calculation of its energy has also been developed.

The great advantage of the computational approach is that it can be applied equally well to the study of the pre-catalyst, resting state and stable (or experimentally observable) catalytic intermediates, but also species that are experimentally inaccessible, such as high energy intermediates and transition states. Being located on an energy maximum, a transition state has no lifetime and can never be observed experimentally - only its relative energy can be accessed by temperature-dependent kinetics studies and this is limited to the ratedetermining step. Calculations, on the other hand, can provide information on both geometry and energy of all transition state. The typical approach of a mechanistic investigation is to gather as much experimental information as possible, revealing the nature, structure and relative energy of the low-energy intermediates and possibly the relative energy of the TS of the RDS ( $\Delta G^{\ddagger}$ cycle). Then the computational approach can fill the gaps, with the calculation of any unobserved intermediate and all the transition states. When more than one mechanistic hypothesis withstands the challenge of all experimental tests, then computational studies can help discarding those that are associated with higher $\Delta G^{\ddagger}$ cycle.

Computational studies, like the experimental ones, are not devoid of complications. There are many choices to be made when embarking into a computational study, all involving a certain number of necessary approximations to make the calculation feasible or less time consuming. The more accurate methods entail a greater computational cost and one must find the best compromise allowing the maximum computational precision in a reasonable timescale. Grossly speaking, there are three types of approximations: (a) those associated with 
the computational method; $(b)$ those associated with the basis functions used to run the calculations; $(c)$ those associated with the chosen chemical model.

\subsection{Computational methods}

The simplest methods available are those based on molecular mechanics (also termed force field methods). They have a convenient scaling of the calculation with the size of the system $\left(N^{2}\right.$ where $N$ is the number of atoms) and can therefore be easily applied to very large molecules. They provide useful information on the most favourable conformation of a molecule, but they are very imprecise in terms of total energy and have limited applicability to describe "unconventional" bonding situations, as found for instance in transition states. In addition, the quality of the results obtained for molecules containing transition metals are less satisfactory than those obtained for organic molecules. Therefore, these methods are generally not used in the study of catalytic mechanisms, except in mixed approaches in combination with quantum mechanical methods (QM/MM, vide infra).

All other methods are based on quantum mechanics and are associated to either molecular orbital (MO) theory or density functional theory (DFT). Both need atomic wavefunctions (orbitals) as input, knowledge of how many electrons are present in the system, knowledge of the overall spin multiplicity, and a starting guess structure. The simplest and less computationally intensive MO methods are the so-called semi-empirical methods, where empirical values replace the 4-center coulombic and exchange integrals that otherwise require the major portion of the computational time. As a result, the calculations scale only as $N^{2}$ or $N^{3}$ ( $N$ being the number of atomic wavefunctions used), depending on the method used, see Table 3. With the increase of computational power and the advent of user-friendly software packages for quantum mechanical calculations, these methods have fallen out of fashion, given their low precision. 
Table 3. Scaling properties of different quantum chemical computations.

\begin{tabular}{ll}
\hline Method & Scaling \\
\hline Semi-empirical & $N^{2}-N^{3}$ \\
DFT & $N^{3}$ \\
HF, MCSCF, CASSCF & $N^{4}$ \\
MP2 & $N^{5}$ \\
MP3, CISD, CCSD, QCISD & $N^{6}$ \\
MP4, CCSD(T), QCISD(T) & $N^{7}$ \\
MP5, CISDT, CCSDT & $N^{8}$ \\
MP6 & $N^{9}$
\end{tabular}

${ }^{\mathrm{a}} N$ is the number of atomic wavefunctions (basis functions) used in the calculation.

The so-called $a b$ initio methods are QM methods based on MO theory where all needed values are computed with the sole knowledge of the molecular geometry, atomic wavefunctions to be employed (see next section), number of electrons and spin state. They are based on the monoelectronic approximation whereby the electron-electron repulsive terms in the Hamiltonian are replaced by an effective "average potential" in which each electron moves. Since the form of this average potential depends on the solution itself, the calculation is iterative, known as Self Consistent Field (SCF), converging when the output density matrix is identical to that used as input. The simplest of these methods is the Hartree-Fock (HF) method, which uses a single electronic configuration (single Slater determinant) to describe the electronic structure. In simple terms, this means that the solution is a unique set of molecular orbitals that are then filled with the available electrons using the aufbau principle to give a single configuration with the required spin. This method scales as $N^{4}$, because of the need to calculate all 4-center integrals. It provides much better results than the semi-empirical methods, in particular in terms of the optimized geometries. However, the energies are affected by large errors and this level of theory is unsuitable for accurate studies of energy 
profiles, as in a catalytic mechanism. The major problem of this method is the neglect of electronic correlation, which is a consequence of the monoelectronic approximation.

$A b$ initio methods that recover, at least partially, the effects of electronic correlation are much more computer-intensive. Only a list of the most common methods and a cursory description of their philosophy is given here. More details can be found in specialized sources. ${ }^{[31,32]}$ Multiple-configuration SCF (MCSCF) allows the global state function to be described by a combination of more than one Slater determinant (i.e. configuration), each with its own separately optimized MO's. Thus, the MO's do not have unique energy and the electronic structure is defined in terms of the occupation numbers. This method is useful for molecular ground states that are quasi-degenerate with low-lying excited states or in bond breaking situations. The configurations are judiciously chosen by varying the occupation of a certain number $n$ of orbitals in the frontier region with a certain number $m$ of electrons. This is the so-called active space, indicated as $(m, n)$. This method takes well into account static correlation, but not dynamic correlation (i.e. the correlation associated with the movement of electrons). The complete active space self-consistent field (CASSCF), once the active space $(m, n)$ is selected, uses all possible arrangements of the $m$ electrons in the $n$ orbitals. The scaling is still as $N^{4}$, but the computational time increases with the number of determinants. An even more sophisticated method is based on the Complete Active Space Perturbation Theory (CASPT2), involving a second order perturbation treatment of each configuration generated by the CASSCF calculation. This introduces suitable consideration of the dynamic part of the electronic correlation and results in rather accurate calculations, albeit it is rather time consuming.

Configuration interaction (CI) methods use a single HF determinant, thus the MO's have unique energies, but allow mixing with excited states obtained by promoting electrons from low-energy filled to higher-energy empty orbitals. CISD, CISDT, etc. indicate methods 
using only single-double, or single-double-triple excitations to generate the new configurations. Truncation of the virtual space is very important to save computational time, since this method scales as $N^{6}$ or higher, depending on the type of excitations used. Contrary to MCSCF, these methods do not work well for nearly degenerate ground states, but take better into account the dynamic correlation. Full CI is a CI method where the active space is all electrons and all orbitals. It is considered as the "exact" solution within the chosen basis set, but even nowadays this is at reach only for the smallest molecules.

Coupled-cluster theory is similar in formalism to CI but more powerful (the higher excitations are generated by a series expansion from simpler excitations). The nomenclature is the same, with CCSD, CCSDT etc. $\operatorname{CCSD}(\mathrm{T})$ means that the triple excitations are only treated at the perturbative level. QCI means quadratic $C I$ and it is an extension of configuration interaction that corrects for size-consistency errors. It gives comparable results to the coupled-cluster method.

Finally for methods based on MO theory, the Møller-Plesset approach uses perturbation theory at the level $n(\mathrm{MPn})$, where the perturbation term is the correlation potential. Calculations up to MP4 are available in most computer packages. Higher level ones are possible but rarely used because of their computational cost and because studies have shown that the method is not necessarily convergent at higher orders. Like CI calculations, this approach has limitations for nearly degenerate ground states.

DFT methods ${ }^{[33]}$ have developed more recently and have quickly gained the favours of the computational chemists for a relatively simple reason: they scale only as $N^{3}$ (except for hybrid methods, going as $N^{4}$ ) and provide results in many cases of comparable quality or better than the correlated ab initio methods such as MP2. Electronic correlation is in fact taken into account by the DFT methods. In this theory, the energy is a functional which depends on the density function and among its contributions - kinetic, electron-nucleus 
attraction, electron-electron repulsion, and exchange-correlation - the expression of the latter $\left[E_{x c}(\rho)\right]$ is unknown. Thus, approximations are needed. Contrary to the ab initio non perturbative methods, this approach does not fulfil the variational theorem - the calculated energy may be either higher or lower than the true value. This highlights one of the major problems of the DFT methods: no systematic method for improving $E_{x c}(\rho)$ exists.

Like for ab initio methods, several levels of this theory exist. The simplest is the local density approximation (LDA), where the energy density in a given position is assumed to depend only on the density value at that position. The LDA methods tend to yield too high energies for the HOMO (thus, too low ionization potentials) and a poor description of electron-rich species such as anions. Methods based on the generalized gradient approximation (GGA), also called "gradient-corrected" LDA, define an energy density function which depends not only on the local value of the density, but also on its gradient (local extent of change). A great many GGA functionals have been developed separately for the exchange part and for the correlation part. The most popular are the B functional (Becke, $1988)^{[34]}$ for exchange and the P86 (Perdew, 1986) ${ }^{[35]}$ LYP (Lee, Young and Parr, 1988), ${ }^{[36]}$ and PW91 (Perdew and Wang, 1992) ${ }^{[37]}$ functional for correlation, but new ones keep adding to the list. These functionals include one or more empirical parameter(s) the value(s) of which is(are) optimized by fitting to the exactly known exchange energies for a number of elements.

The use of more parameters results in significant improvements, at the cost of incorporating a portion of the exact HF exchange, and leads to the so-called hybrid functionals. The exact exchange energy functional is expressed in terms of the orbitals rather than the density, so it is termed an implicit density functional. Hybridization involves only the exchange part of the functional, which is then used together with the above mentioned correlation functionals, leading to popular exchange-correlation functionals such as B3LYP, B3PW91, etc. 
As already stated, DFT provides results of comparable quality to ab initio correlated methods at significantly lower computational cost. In general, DFT does not perform as well as ab initio methods for the computation of weak interactions (H-bonds, van der Waals, charge transfer, etc.), but new functionals with improved performances in those situations have been proposed. ${ }^{[38]}$ As stated above, the major problem of DFT is that there is no obvious way to determine a priori which functional may be optimal for a particular case of interest, since there is no variational principle assisting with the evaluation of the computational accuracy. ${ }^{[39]}$ Thus, it is absolutely necessary to recur to benchmarking, which can be carried out in one of two ways. The best one is to compare the calculations with the experimental data, when available. In terms of geometries, this is sometimes possible for stable compounds when these can be isolated and structurally characterized. It is never possible for transition states. In terms of relative energies, these are not always easily accessible from calorimetric or temperature-dependent equilibrium studies if dealing with thermodynamic parameters, or from temperature dependent kinetics studies if dealing with activation barriers. The second way is to compare the performance of various functionals in DFT with the performance of a high-level ab initio method, considered to yield the "exact" energy (e.g. full CI or CCSDT), for a computationally accessible simplified model related to the system of interest.

One common problem in computational chemistry, of particular interest in homogeneous catalysis, is the accurate handling of large size molecules where the most important energetic effects are caused by changes in chemical bonding in a limited portion of the molecule (the metal coordination sphere), while weak non covalent interactions in the ligand periphery fine tune the barrier height (activity) as well as the relative barrier heights of competitive pathways (selectivity). Treatment of such systems entirely at the quantum mechanical (QM) level would be too costly, whereas treatment at a lower level (semiempirical, or molecular mechanics (MM)) is unsatisfactory. Thus, mixed methods have been 
developed. The QM/MM method consists of separating the molecule into two parts where one, where the bonds are being broken and formed and where electronic effects on the reactive bonds are strong, is handled at the QM level, while the rest is handled at the MM level. This approach combines the strength of both QM accuracy and MM speed, and its use in the investigation of catalytic mechanisms is growing. ${ }^{[40]}$ Methods based on the same principle but allowing multiple layers, each handled at a different level of theory, have also been developed, a popular one being ONIOM. ${ }^{[41]}$

\subsection{Basis functions}

All quantum mechanical methods need wavefunctions describing the orbitals of the participating atoms as input (the so-called basis set). There are two decisions to be made in the choice of a basis set: $(a)$ the number of functions in the basis set; $(b)$ their mathematical representation (orbital shape) for use in the calculations.

Within each computational approach, the "exact" solution requires a complete basis set (all possible atomic orbitals for each atom, whether occupied or empty - an infinite number), which is computationally impossible. The problem is to decide where to truncate the basis set in order to reduce the computational cost (reminder: scaling goes as a power of the number $N$ of basis functions) while keeping a satisfactory description of the molecular orbitals (for MO methods) or density (for DFT methods). The minimum number of basis functions needed in order to obtain a chemically reasonable representation (minimum basis set) is made up of all the occupied atomic orbitals in the separate atoms. For instance, a $3 d$ atom such as $\mathrm{Mn}$ or Fe would be described using the $1 \mathrm{~s}, 2 \mathrm{~s}, 2 \mathrm{p}, 3 \mathrm{~s}, 3 \mathrm{p}$, and $3 \mathrm{~d}$ orbitals. This is seldom satisfactory for the accurate calculation of geometries and energies, the main reason being that the electron density of atoms is distorted (polarized) upon formation of the chemical bonds. To take this phenomenon into account, it is necessary to add polarization functions, which are unoccupied 
atomic orbitals with one additional angular node (higher $l$ value) relative to the bonding orbitals (e.g. $p$ functions to polarize $s$ electrons, $d$ functions for $p$ electrons, $f$ functions for $d$ electrons etc.). Typical basis sets do not include virtual orbitals from higher-order shells, except for the polarization functions.

Once the number of atomic orbitals is defined, it is necessary to chose the mathematical representation for the wavefunction, which is most conveniently defined in polar coordinates, $\Psi(\mathrm{r}, \theta, \phi)$. The problem is in fact limited to the radial component $\mathrm{R}_{\mathrm{nl}}(\mathrm{r})$ of the function, see equation 5, because the possible angular components $\mathrm{Y}_{\operatorname{lm}}(\theta, \phi)$ (the so-called spherical harmonics) are know exactly. Two main forms of the radial component are used: the Slatertype orbitals (STO) and the Gaussian-type orbitals (GTO).

$$
\Psi(\mathrm{r}, \theta, \phi)=\mathrm{R}_{\mathrm{n}}(\mathrm{r}) \cdot \mathrm{Y}_{\operatorname{lm}}(\theta, \phi)
$$

The STO's have the general form $\mathrm{r}^{\mathrm{n}-1} \mathrm{e}^{-\zeta \mathrm{r}}$ which gives a correct radial trend, notably the exponential decay and a cusp at the origin. They have, however, the great disadvantage of not giving analytical solutions to the four-index integrals, thus imposing a great computational effort for their calculation by numerical methods. These functions are commonly used in combination with the semi-empirical methods and with the DFT methods (except those using hybrid functionals), which do not compute the four-index integrals. The GTO's, of type $\mathrm{r}^{\mathrm{n}-1} \mathrm{e}^{-\alpha \mathrm{r}^{2}}$, have the advantage of yielding analytical solutions for the four-index integrals, with speedup by 4-5 orders of magnitude compared to STO's, but do not correctly reproduce the behaviour close to the nucleus (where the electron density is maximum) and decay to zero too quickly, see Figure 21a. However, a better Gaussian-type function can convenient be constructed as a linear combination of GTO's with different exponential coefficients. For instance, Figure $21 b$ shows the result of combining three Gaussian functions with loose, 
medium and tight radial dependence into a single sum, mimicking the shape of an STO. The nomenclature STO-3G is used for this type of linear combination. When the relative coefficients in these linear combinations are kept fixed during the SCF procedure, the functions take the name of contractions.

(a)

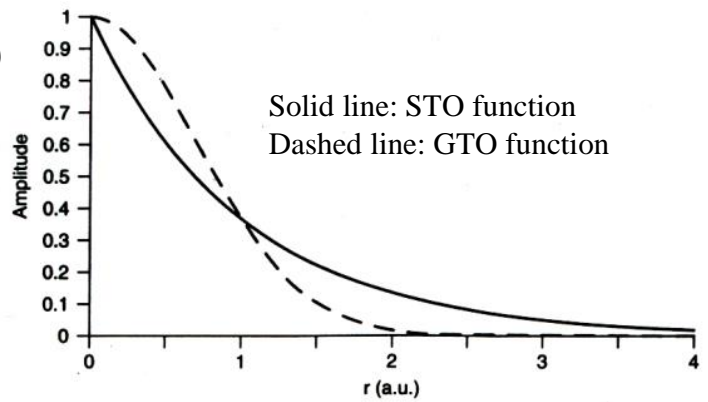

(b)

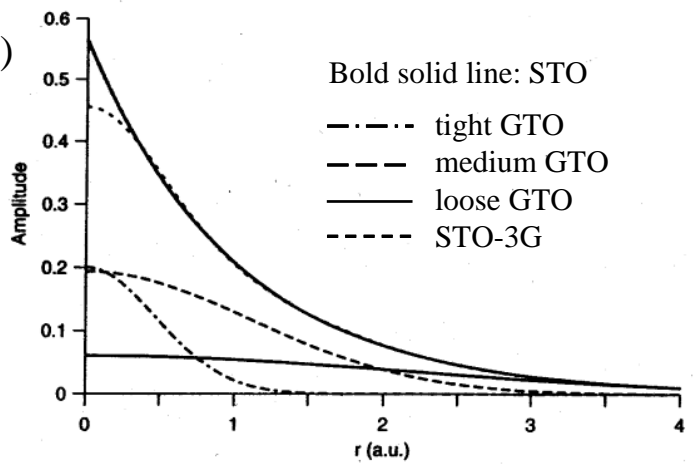

Figure 21. Shape of $n=0$ (no node) STO and GTO functions.

Additional flexibility and better results are obtained when the coefficients of the different GTO components (also called primitives) of a linear combination are allowed to be independently optimized during the SCF procedure. This operation of releasing the constraint between different primitives in a contraction is called a decontraction and the resulting function is called double-zeta if it is the sum of two contractions (each with a variable coefficient but still fixed parameters within each residual contraction), triple-zeta if it is the sum of three contractions, and so forth. Decontracting an atomic orbital function increases its flexibility but introduces additional parameters to be optimized and therefore increases the computational time. 
Certain basis sets use different contraction strategies for different orbitals, depending on whether they are in a core or in a valence shell. This is the philosophy of the split-valence basis sets. It is best to have more flexibility in valence orbitals, better adjusting to the electronic changes associated to chemical bond formation, whereas core orbitals are best described by a single contraction, though with a greater number of primitives in order to better describe electron density that is closer on average to the cusp at the nucleus. The nomenclature is simple to understand: for instance $3-21 \mathrm{G}$ is a split valence using a single contraction with 3 primitives in the core and a double-zeta having two primitives in one contraction and one primitive in the other one; 6-311G has one contraction with 6 primitives in the core and a triple-zeta with 3,1 , and 1 primitives in the three components of the decontracted valence functions. One additional need is presented by atoms that are expected to carry a relatively large electron density, such as electronegative atoms in very polarizable bonds $(\mathrm{C}=\mathrm{O}, \mathrm{C} \equiv \mathrm{N})$ or in anions $\left(\mathrm{RO}^{-}, \mathrm{PF}_{6}^{-}, \mathrm{NiCl}_{4}{ }^{2-}\right.$, etc. $)$, thus giving rise to a relatively diffuse electron density in the resulting molecule. In those cases the valence atomic orbitals are better described by adding an additional very loose primitive (i.e. with a small exponential coefficient), which is termed diffuse function.

A final feature of importance is the effective core potential (ECP), also termed pseudopotential. This is a fixed function describing the totality of the core electrons that are 'frozen', being considered together with the nucleus as a rigid non-polarizable ion core. The greatest benefit associated to the introduction of an ECP is in the reduction of the basis set size, thus the computational time, and in the number of electrons explicitly considered in the calculation, but is also a means to introduce relativistic and other effects. The use of an ECP obviously introduces an additional approximation and is usually implemented only for elements of the $4^{\text {th }}$ shell and heavier, where the motivation is the greatest in terms of computational savings. The two most common approaches associated to the use of an ECP 
are known as 'large core' and 'small core'. The large-core approach is the most drastic simplification, consisting of representing all electrons except those in the valence shell by a pseudopotential. In the small-core ECP approach, the cutoff between core and valence is scaled back by one shell, though several options are possible by selectively including lowershell orbitals of specific angular type. As an example, for an element of the $6^{\text {th }}$ shell such as $W$, with the configuration $(1 s)^{2}(2 s)^{2}(2 p)^{6}(3 s)^{2}(3 p)^{6}(3 d)^{10}(4 s)^{2}(4 p)^{6}(4 d)^{10}(4 f)^{14}(5 s)^{2}(5 p)^{6}(5 d)^{6}$ in a prepared valence state and with the $5^{\text {th }}$ shell $\mathrm{d}$ orbitals also participating in bonding, the "large core ECP" includes shells 1-4, with only the 5s, 5p, 5d, 6s and 6p orbitals (plus perhaps $f$-type polarization functions) treated explicitly in the calculations, whereas a "small core" version may also include the $4 \mathrm{~s}$ and $4 \mathrm{p}$ functions as valence. For the lanthanide elements with configuration $(1 s)^{2}(2 s)^{2}(2 p)^{6}(3 s)^{2}(3 p)^{6}(3 d)^{10}(4 s)^{2}(4 p)^{6}(4 d)^{10}(4 f)^{x}(5 s)^{2}(5 p)^{6}(5 d)^{y}$, the large core ECP would be the same as for $\mathrm{W}$, whereas a small core version may also include the incompletely filled $4 \mathrm{f}$ shell. In many cases results are of sufficiently good quality when using a large core, but in other cases the implicit treatment of the lower shell electrons has shown advantages.

The judicious choice of a basis set, ${ }^{[42,43]}$ together with that of the computational method, is one of the most important preliminaries before a successful calculation can be carried out. Basis sets of all sorts and qualities are included in the library of the available computational software, with or without polarization functions, diffuse functions, ECP etc. Others are downloadable from the Internet ${ }^{[44]}$ or can be obtained from the supporting information of the original research papers.

\subsection{Thermodynamic properties, chemical and solvent model}

When computers were not as fast as they are today, it was standard to simplify the molecular geometry in order to reduce the number of electrons and wavefunctions. Thus 
molecules such as $\mathrm{RhCl}\left(\mathrm{PPh}_{3}\right)_{3}$ would be modelled by $\mathrm{RhCl}\left(\mathrm{PH}_{3}\right)_{3}, \mathrm{Cp}_{2}{ }_{2} \mathrm{WEt}_{2}$ by $\mathrm{Cp}_{2} \mathrm{WMe}_{2}$ (or $\mathrm{Cp}_{2} \mathrm{WH}_{2}$ ), and so forth, providing indicative information on the structure and bonding of the full system but none whatsoever on the energetics. The present computational power allows handling most catalytic systems (containing one or a small number of metals and up to several tens of atoms for a few hundreds of electrons) without simplification except possibly for using mixed QM/MM methods, at relatively accurate levels (DFT or low level ab initio methods). However, obtaining relevant energetic information for the investigation of catalytic mechanisms requires also the accurate modelling of the temperature and solvent effects (both non specific and specific interactions). The standard calculations of molecular electronic structures are carried out on isolated molecules, giving results that are pertinent to the gas phase at zero pressure and zero temperature. Catalytic cycles, on the other hand, involve equilibria and reaction barriers that are related to free energy differences in a condensed phase at elevated temperatures (typically $300-400 \mathrm{~K}$ ). In fact, the calculated energies do not even pertain to the gas-phase at $0 \mathrm{~K}$, because they only deal with the electronic part of the system, the nuclear positions being kept fixed at the equilibrium geometry. The resulting energy value is the electronic energy, $E_{\mathrm{el}}$. The calculation of the total energy at $0 \mathrm{~K}$ needs addition of the nuclear part, which is the ZPVE already mentioned in section 4.1. This correction requires the solution of the nuclear part of the Schrödinger equation, giving all the $3 \mathrm{~N}-6$ vibrational frequencies $v$. At higher temperatures, higher vibrational states also become thermally populated according to the Boltzmann factor. Knowledge of all vibrational frequencies allows the calculation of $\mathrm{E}$ at any temperature. This thermal correction is usually based on the harmonic oscillator approximation (of limited validity for low-frequency vibrations and relatively high temperatures). Further converting this energy value to free energy requires calculation of the PV and TS terms. The PV term is approximated by $k \mathrm{~T}$ on the basis of the ideal gas approximation, whereas $\mathrm{S}$ can be calculated once again from the vibrational 
frequencies through use of the partition function, on the basis of statistical thermodynamics relationships. The various corrections needed to convert the electronic energy into the free energy at the temperature $\mathrm{T}$ are summarized in Figure 22. Obviously, all these values do not correspond to absolute thermodynamic parameters; they are differences relative to the collection of the separate atoms. Thus, the various corrections must also be carried out on the separate atoms. This way, we can obtain gas-phase free energies for the molecule at any temperature, within the limits of the approximations (harmonic oscillator, ideal gas, and the limitations of the computational method and basis set described above). However, the catalyzed transformation occurs in a condensed phase. The question then becomes how to take the solvent effects into account.

$$
E_{\text {el. }} \stackrel{\text { ZPVE }}{\longrightarrow} E_{0 K} \stackrel{\text { Thermal }}{\longrightarrow} E_{\mathrm{T}} \stackrel{k T}{\longrightarrow} H_{\mathrm{T}} \stackrel{-\mathrm{TS}}{\longrightarrow} G_{\mathrm{T}}
$$

Figure 22. The various corrections for transforming the electronic energy into free energy.

In the old days, modelling the solvent environment could not be done and one had to be satisfied with the gas-phase values. This turns out to give reasonable indications, limited to energy and enthalpy, of the real situation in solution provided that the solvent is apolar (e.g. a hydrocarbon, especially a saturated one). The polarity of solvents, on the other hand, change significantly the solution energetics relative to the gas phase because of significant solventsolute interactions that affect the energy/enthalpy terms (for the entropy, vide infra). These interactions are already significant for solutes with low or zero dipole moment but high polarizability, greater for dipolar solutes, and greater still for solutes carrying a charge.

In order to properly take into account the solvent-solute interactions, a calculation of the solute surrounded by a large number of solvent molecules would be necessary, but the computational cost would then become prohibitively high. Instead, the solvent effect is 
modelled as a polarizable continuum (PCM, standing for polarizable continuum model). ${ }^{[45]}$ Many different variations of the basic PCM are now available in software packages. A popular one is the Conductor PCM (C-PCM) ${ }^{[46]}$ which has the advantage to be applicable to large and irregularly formed molecular structures and is more accurate for solvents with a higher permittivity (e.g. water). All these models can be used for calculating energies and gradients at the Hartree-Fock and density functional theory (DFT) levels. In these models, the solvent free energy change is computed as the sum of three terms: electrostatic, dispersionrepulsion, and cavitation, the last being related to the creation of the cavity to be occupied by the solute molecule. A solute polarization energy term is also included. The cavitation term is quite important for the calculation of the solvation correction and the way in which the solute geometry is used to calculate the cavity volume and shape is particularly critical.

It is important to underline that the correction term obtained from this calculation, usually referred to as $\Delta G_{\text {solv }}$, represents a free energy change for the solvent, not for the solute for which only a corrective term for the polarization energy related to the solvent-solute interaction is taken into account. The obvious question is how to apply this correction and what is the meaning of the resulting number? In principle, the corrective term can be added to any of the gas phase values shown in Figure 22. For instance, application to the electronic energy gives a solvated electronic energy, $E_{\mathrm{el}, \text { solv. }}$, application to the enthalpy gives a solvated enthalpy, $H_{\mathrm{T} \text {,solv. }}$ and so forth, as shown in Figure 23. However, none of these values represents anything real, because the molecular geometry may be expected to slightly change on going from the gas phase to the solution. Ideally, the molecular geometry should be optimized in the presence of the solvent model. The calculation of the vibrational frequencies should also be carried out in the presence of the solvent model. It is a well established experimental fact that vibrational frequencies are solvent dependent, thus applying a thermal and entropy correction in a condensed phase by using gas-phase frequencies is not ideal. 
However, though possible, geometry optimizations and frequency calculations in the presence of a solvent model are often rather tricky to converge. Therefore, even though entailing yet another approximation, the most popular approach is to apply the solvation correction to the gas-phase energetic parameters.

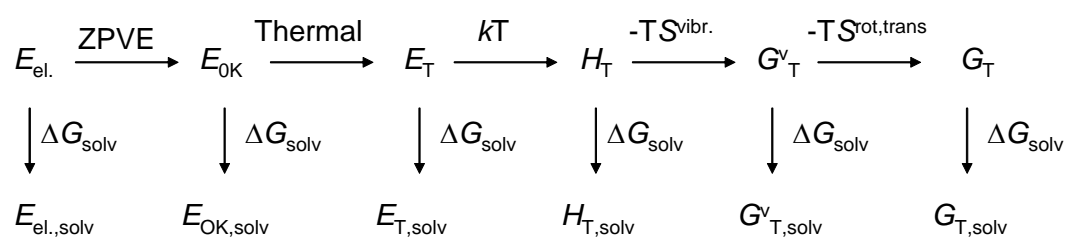

Figure 23. Application of the solvation energy correction to gas phase parameters.

There is yet another problem affecting the estimation of solute free energies, related to the entropic term and how it changes upon going from the gas phase to the solution. The solute loses much of its translational and rotational entropy, since these degrees of freedom, though not totally quenched, are transformed into low frequency "librations" in the solvent cage. A fundamental problem is that no method exists to calculate precisely how much of this gas-phase entropy, which is a significant proportion of the total, is lost upon going to the solution. This leads to different approaches for the evaluation and subsequent application of the solution energetic properties. In one relatively popular approach, $\Delta G_{\text {solv }}$ is applied to the gas-phase energy or enthalpy, to obtain the corresponding solution values and these are treated as such. They can therefore be compared with the experimentally available energy or enthalpy (not free energy!) changes. Many users tend to confuse the term obtained as just described as a solute free energy since the computer yields a free energy term, $\Delta G_{\text {solv }}$, but in fact the solute has no TS correction. A second approach is to apply the solvation energy correction to $G_{\mathrm{T}}$. The result is a $G_{\mathrm{T}, \text { solv }}$ value, but the question is the overestimation of the solute translational and rotational entropy. A third approach consists of neglecting completely 
the translational and rotational contributions to the entropy, and applying only the $\mathrm{T} S^{\text {vibr }}$ correction ( $S^{\mathrm{vibr}}=$ vibrational entropy) and the solvation correction to the gas-phase enthalpy, obtaining a value than can be termed $G^{\mathrm{v}}$ T,solv. However, even this value does not give a correct representation of the solute free energy. Published computational studies of catalytic cycles have used anyone of these three approaches but none is ideal. To our knowledge, there is at the moment no universally accepted model for to accurate estimation of a solute free energy in solution. For reactions involving the same number of molecules on the reactant and product sides, the thermodynamic changes calculated with any of the above models are reasonably similar because the errors in handling the translational and rotational entropy tend to cancel out. All the models also give similar results for the activation barriers when the activation entropy ( $\Delta S_{\text {cycle }}$ for a catalytic process) is small. However, great care must be exercised for reactions involving a large change in the number of species. This is especially true when a charge separation or annihilation is involved between the resting state and the TS(RDS), because of possible equilibria between the free ions and ions pairs. Indeed, charged species interact strongly not only with solvent molecules but also with the counterion, including the possible establishment of specific interactions (H-bonding, $\pi$-stacking, etc.). In these cases it is necessary to include the counterion explicitly in the calculation, a seldom occurrence in computational studies.

As a last remark, use of a PCM to represent solvent effects has serious limitations when the solvent also establishes specific interactions with the solute, such as hydrogen bonds or coordinative bonds. In such cases, the explicit inclusion of a suitable number of solvent molecules, in addition to the PCM, becomes necessary in order to obtain meaningful results.

\section{CONCLUSION}


The full elucidation of a catalytic mechanism is one of the greatest challenges for a research chemist. Each catalytic transformation has its own specificity and no single approach exists, valid in all cases, to provide the correct solution. However, many approaches are available, as shown in this article. Each one of them can illuminate a particular aspect of the mechanism, such as the structure of the resting state, or the relative energy of the transition state of the rate-determining step, or again a structural feature in a given intermediate that determines the catalyst selectivity. Computational methods have now reached a level of speed and precision where they can really assist the experimental chemist in the comparison of alternative pathways or in elucidating the structural features of key transition states. The best way to tackle the problem is to adopt as many different approaches as possible in order to arrive at the validation of one mechanistic hypothesis while hopefully discarding all the others. Only then can we feel reasonably confident about our knowledge of the catalytic mechanism.

\section{ACKNOWLEDGEMENT}

I am grateful to the Centre National de la Recherche Scientifique, to the Institut National Polytechnique de Toulouse and to the Institut Universitaire de France for providing facilities and research funding. I am also grateful to the Centre Interuniversitaire de Calcul de Toulouse (project CALMIP) for granting free computational time.

\section{REFERENCES}

[1] B. Cornils and W. A. Herrmann, Applied homogeneous Catalysis with Organometallic Compounds: A Comprehensive Handbook, 2nd Ed., Wiley -VCH, Weinheim, 2002, pp.

[2] P. W. N. Van Leeuwen, Homogeneous Catalysis. Understanding the Art, Kluwer Academic Publishers, Dordrecht, The Netherlands, 2004, pp.

[3] R. I. Masel, Chemical Kinetics and Catalysis, John Wiley \& Sons, Inc., New York, 2001, pp. 
[4] F. Maseras and A. Lledós, Computational Modeling of Homogeneous Catalysis, Kluwer Academic Publishers, Dordrecht, The Netherlands, 2002, pp.

[5] B. T. Heaton in Mechanisms in Homogeneous Catalysis. A Spectroscopic Approach, Vol. (Ed.: Wiley-VCH, Weinheim, 2005, pp.

[6] K. Morokuma and D. G. Musaev in Computational Modeling for Homogeneous and Enzymatic Catalysis: A Knowledge-Base for Designing Efficient Catalysts, Vol. (Ed.: Wiley-VCH, Weinheim, 2008, pp.

[7] D. Heller, H. J. Drexler, J. S. You, W. Baumann, K. Drauz, H. P. Krimmer and A. Borner, Chem. Eur. J. 2002, 8, 5196-5203.

[8] M. Garland and P. Pino, Organometallics 1991, 10, 1693-1704.

[9] P. W. N. M. Van Leeuwen and C. Claver, Rhodium Catalyzed Hydroformylation, Kluwer Academic Publishers, Dordrecht, The Netherlands, 2000, pp.

[10] X. F. Wu, J. K. Liu, D. Di Tommaso, J. A. Iggo, C. R. A. Catlow, J. Bacsa and J. L. Xiao, Chem. Eur. J. 2008, 14, 7699-7715.

[11] D. A. Singleton and A. A. Thomas, J. Am. Chem. Soc. 1995, 117, 9357-9358.

[12] L. K. Vo and D. A. Singleton, Org. Lett. 2004, 6, $2469-2472$.

[13] U. Nettekoven and J. F. Hartwig, J. Am. Chem. Soc. 2002, 124, 1166-1167.

[14] M. H. O'leary, Methods Enzymol 1980, 64, 83-104.

[15] O. Pàmies and J. E. Bäckvall, Chem. Eur. J. 2001, 7, 5052-5058.

[16] J.-J. Brunet, N.-C. Chu and M. Rodriguez-Zubiri, Eur. J. Inorg. Chem. 2007, 47114722.

[17] A. L. Casalnuovo, J. C. Calabrese and D. Milstein, J. Am. Chem. Soc. 1988, 110, 6738-6744.

[18] R. Dorta, P. Egli, F. Zurcher and A. Togni, J. Am. Chem. Soc. 1997, 119, 10857-10858.

[19] J. Zhou and J. F. Hartwig, J. Am. Chem. Soc. 2008, 130, 12220-12221.

[20] J. Andrieu, J. M. Camus, C. Balan and R. Poli, Eur. J. Inorg. Chem. 2006, 62-68.

[21] C. R. Landis and J. Halpern, J. Am. Chem. Soc. 1987, 109, 1746-1754.

[22] A. S. C. Chan, J. J. Pluth and J. Halpern, Inorg. Chim. Acta 1979, 37, L477-L479.

[23] L. Damoense, M. Datt, M. Green and C. Steenkamp, Coord. Chem. Rev. 2004, 248, 2393-2407.

[24] R. Eisenberg, Acc. Chem. Res. 1991, 24, 110-116.

[25] S. B. Duckett, C. L. Newell and R. Eisenberg, J. Am. Chem. Soc. 1994, 116, 1054810556.

[26] E. Fernandez, A. Ruiz, C. Claver, S. Castillon, A. Polo, J. F. Piniella and A. AlvarezLarena, Organometallics 1998, 17, 2857-2864.

[27] A. S. C. Chan and J. Halpern, J. Am. Chem. Soc. 1980, 102, 838-840.

[28] R. Giemoth, H. Heinrich, N. J. Adams, R. J. Deeth, J. Bargon and J. M. Brown, J. Am. Chem. Soc. 2000, 122, 12381-12382.

[29] L. A. Van Der Veen, M. D. K. Boele, F. R. Bregman, P. C. J. Kamer, P. W. N. M. Van Leeuwen, K. Goubitz, J. Fraanje, H. Schenk and C. Bo, J. Am. Chem. Soc. 1998, 120, 11616-11626.

[30] R. Poli and J. N. Harvey, Chem. Soc. Rev. 2003, 32, 1-8.

[31] C. J. Cramer, Essentials of Computational Chemistry, John Wiley \& Sons, Chichester, 2002, pp.

[32] F. Jensen, Introduction to Computational Chemistry, John Wiley and Sons, Chichester, England, 2007, pp.

[33] R. G. Parr and W. Yang, Density-Functional Theory of Atoms and Molecules, Oxford University Press, Oxford, 1994, pp.

[34] A. D. Becke, Phys. Rev. A 1988, 1988, 3098-3100.

[35] J. P. Perdew, Physical Review B-Condensed Matter 1986, 33, 8822-8824.

[36] C. T. Lee, W. T. Yang and R. G. Parr, Phys. Rev. B 1988, 37, 785-789.

[37] J. P. Perdew and Y. Wang, Phys. Rev. B 1992, 45, 13244-13249.

[38] Y. Zhao and D. G. Truhlar, Theor. Chem. Acc. 2008, 120, 215-241.

[39] S. F. Sousa, P. A. Fernandes and M. J. Ramos, J. Phys. Chem. A 2007, 111, 1043910452. 
Running title: Mechanisms in Homogeneous Catalysis

[40] C. Bo and F. Maseras, Dalton Trans. 2008, 2911-2919.

[41] R. D. J. Froese and K. Morokuma in Encylopedia of Computational Chemistry, Vol. 2 (Ed.: P. v. R. Schleyer), John Wiley and Sons, Chichester, 1998, pp. 1244-1257.

[42] E. R. Davidson and D. Feller, Chem. Rev. 1986, 86, 681-696.

[43] D. Feller, J. Comp. Chem. 1996, 17, 1571-1586.

[44] https://bse.pnl.gov/bse/portal.

[45] J. Tomasi and M. Persico, Chem. Rev. 1994, 94, 2027-2094.

[46] V. Barone and M. Cossi, J. Phys. Chem. A 1998, 102, 1995-2001. 